\title{
Management of hospitalised adults with coronavirus disease 2019 (COVID-19): a European Respiratory Society living guideline
}

\author{
James D. Chalmers $\mathbb{C}^{1,23}$, Megan L. Crichton ${ }^{1}$, Pieter C. Goeminne ${ }^{2}$, Bin Cao ${ }^{3}$, Marc Humbert $\mathbb{\circledR}^{4}$, \\ Michal Shteinberg $\mathbb{1}^{5}$, Katerina M. Antoniou ${ }^{6}$, Charlotte Suppli Ulrik $\mathbb{B}^{7}$, Helen Parks ${ }^{8}$, Chen Wang ${ }^{9}$, \\ Thomas Vandendriessche ${ }^{10}$, Jieming $\mathrm{Qu}^{11,12}$, Daiana Stolz ${ }^{13,14,15}$, Christopher Brightling ${ }^{16}$, \\ Tobias Welte (17) ${ }^{17}$, Stefano Aliberti ${ }^{18,19}$, Anita K. Simonds ${ }^{20}$, Thomy Tonia ${ }^{21}$ and Nicolas Roche ${ }^{22,23}$
}

\begin{abstract}
${ }^{1}$ School of Medicine, University of Dundee, Dundee, UK. ${ }^{2}$ Department of Respiratory Medicine, AZ Nikolaas, Sint-Niklaas, Belgium. ${ }^{3}$ Department of Respiratory and Critical Care Medicine, Clinical Microbiology and Infectious Disease Lab, China-Japan Friendship Hospital, National Center for Respiratory Medicine, Institute of Respiratory Medicine, Chinese Academy of Medical Science, National Clinical Research Center of Respiratory Diseases, Beijing, China. ${ }^{4}$ Service de Pneumologie et Soins Intensifs, Hôpital Bicêtre, Assistance Publique-Hôpitaux de Paris (AP-HP); Université Paris-Saclay; Inserm UMR_S 999, Le Kremlin Bicêtre, France. ${ }^{5}$ Pulmonology institute and CF Center, Carmel Medical Center and the Technion-Israel Institute of Technology, Haifa, Israel. ${ }^{6}$ Laboratory of Molecular and Cellular Pneumonology, Department of Respiratory Medicine, School of Medicine, University of Crete, Heraklion, Greece. ${ }^{7}$ Department of Respiratory Medicine, Copenhagen University Hospital-Hvidovre Hospital, Hvidovre, Denmark. ${ }^{8}$ European Lung Foundation, Sheffield, UK. ${ }^{9}$ Department of Pulmonary and Critical Care Medicine, Center of Respiratory Medicine, China-Japan Friendship Hospital, Chinese Academy of Medical Sciences \& Peking Union Medical College, National Clinical Research Center of Respiratory Diseases, Beijing, China. ${ }^{10} \mathrm{KU}$ Leuven Libraries - 2Bergen - Learning Centre Désiré Collen, Leuven, Belgium. ${ }^{11}$ Department of Pulmonary and Critical Care Medicine, Ruijin Hospital, Shanghai, China. ${ }^{12}$ Institute of Respiratory Diseases, Shanghai Jiao Tong University School of Medicine, Shanghai, China. ${ }^{13}$ Clinic of Respiratory Medicine and Pulmonary Cell Research, University Hospital Basel, Basel, Switzerland. ${ }^{14}$ Clinic of Respiratory Medicine, Medical Center - University of Freiburg, Freiburg, Germany. ${ }^{15}$ Faculty of Medicine, University of Freiburg, Freiburg, Germany. ${ }^{16}$ Institute for Lung Health, Leicester NIHR BRC, University of Leicester, Leicester, UK. ${ }^{17}$ Medizinische Hochschule Hannover, Direktor der Abteilung Pneumologie, Hannover, Germany. ${ }^{18}$ Department of Biomedical Sciences, Humanitas University, Pieve Emanuele, Milan, Italy. ${ }^{19}$ IRCCS Humanitas Research Hospital, Respiratory Unit, Rozzano, Italy. ${ }^{20}$ Sleep and Ventilation Unit, Royal Brompton and Harefield Hospital, Guys and St Thomas NHS Foundation Trust, London, UK. ${ }^{21}$ Institute of Social and Preventive Medicine, University Bern, Bern, Switzerland. ${ }^{22}$ Respiratory Medicine, Cochin Hospital, APHP Centre-University of Paris, Cochin Institute (INSERM UMR1016), Paris, France. ${ }^{23}$ J.D. Chalmers and N. Roche are task force co-chairs.
\end{abstract}

Corresponding author: James D. Chalmers (jchalmers@dundee.ac.uk)

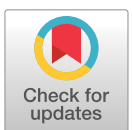

Copyright $\odot$ The authors 2022

This version is distributed under the terms of the Creative Commons Attribution NonCommercial Licence 4.0. For commercial reproduction rights and permissions contact permissions@ersnet.org

Received: 16 April 2022 Accepted: 31 May 2022

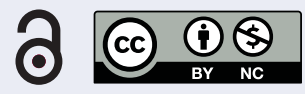

Shareable abstract (@ERSpublications)

The ERS COVID-19 guidelines make recommendations for corticosteroids, anti-IL-6 monoclonal antibodies, baricitinib, anticoagulation and non-invasive respiratory support for hospitalised patients with COVID-19 https://bit.ly/2OlpniF

Cite this article as: Chalmers JD, Crichton ML, Goeminne PC, et al. Management of hospitalised adults with coronavirus disease 2019 (COVID-19): a European Respiratory Society living guideline. Eur Respir J 2021; 57: 2100048 [DOI: 10.1183/13993003.00048-2021].

\section{Abstract}

Introduction Hospitalised patients with coronavirus disease 2019 (COVID-19) as a result of severe acute respiratory syndrome coronavirus 2 (SARS-CoV-2) infection have a high mortality rate and frequently require non-invasive respiratory support or invasive mechanical ventilation. Optimising and standardising management through evidence-based guidelines may improve quality of care and therefore patient outcomes. Methods A task force from the European Respiratory Society, and endorsed by the Chinese Thoracic Society, identified priority interventions (pharmacological and non-pharmacological) for inclusion in this "living guideline" using the PICO (Population, Intervention, Comparator, Outcomes) format. The GRADE (Grading of Recommendations, Assessment, Development and Evaluations) approach was used for assessing the quality of evidence and strength of recommendations. Systematic literature reviews were performed, and data pooled by meta-analysis where possible. Evidence tables were presented and evidence-to-decision frameworks were used to formulate recommendations.

Results Based on the available evidence at the time of guideline update (14 February 2022) the panel makes a strong recommendation in favour of the use of systemic corticosteroids in patients requiring supplementary oxygen or ventilatory support, and of interleukin-6 receptor antagonist monoclonal 
antibodies or baricitinib for patients requiring supplementary oxygen and for the use of anticoagulation in hospitalised patients. The panel makes a conditional recommendation for continuous positive airway pressure in patients with acute hypoxaemic respiratory failure and for combination treatment with casirivimab and imdevimab in patients who have no detectable SARS-CoV-2 spike antibodies (seronegative) and a susceptible variant. No recommendation was made for remdesivir in patients requiring supplemental oxygen. The panel recommended against multiple therapeutics, including hydroxychloroquine, azithromycin, convalescent plasma, lopinavir-ritonavir and colchicine. Further recommendations for research are made.

Conclusion Several interventions reduce mortality and improve clinical outcomes in patients with severe COVID-19 infection. These guidelines will be regularly updated as further evidence becomes available.

Introduction

COVID-19 (coronavirus disease 2019) is the disease resulting from infection by the SARS-CoV-2 (severe acute respiratory syndrome coronavirus 2) virus. First identified in Wuhan, China in December 2019 [1], the disease rapidly developed into a global pandemic with over 287 million infections and more than 5.4 million deaths recorded worldwide as of the end of 2021 [2-4].

The spectrum of disease caused by SARS-CoV-2 infection is remarkably broad, ranging from true asymptomatic or paucisymptomatic infection to fatal acute respiratory distress syndrome [5-8]. Risk factors for hospitalisation and mortality have been defined, including age, male sex, obesity and co-morbid conditions [9-12]. Risk of hospitalisation and mortality is most strongly associated with age, and therefore SARS-CoV-2 infection rarely results in hospitalisation or mortality in children [13].

The epidemiology and clinical presentation of the virus has changed over the course of the pandemic with the emergence of variants which enhance the transmissibility, severity and immune evasion of the virus [14-17]. At the time of writing, globally the dominant variants are the delta variant, which has increased transmissibility and severity compared to its preceding variants, and the omicron variant, which has enhanced transmissibility and immune evasion, allowing it to infect large numbers of previously infected or vaccinated individuals [14-18]. There is evidence that the omicron variant carries a lower risk of hospitalisation compared to preceding variants [19, 20]. Vaccination against SARS-CoV-2 has provided $90-95 \%$ protection against the risk of severe disease and hospitalisation in countries where vaccines have been made available and preliminary evidence suggests vaccine protection against severe disease is maintained against emerging variants [21, 22].

COVID-19 is often described as a biphasic illness with distinct stages [23]. The initial stage of infection, with fever, cough and other symptoms, is associated with the highest viral loads which peak in the first 7 days of illness and the majority of patients will not require hospitalisation [24]. Live virus remains detectable in the respiratory tract for up to 9 days and, in the majority of individuals, symptoms start to improve after the first week [24]. In a proportion of patients, however, a second phase characterised by a dysfunctional host inflammatory response and the development of lung inflammation and lung injury follows [25-29]. Patients experiencing inflammatory lung injury will frequently require hospitalisation. The inflammatory response in moderate and severe COVID-19 has been variously described as a pro-inflammatory cytokine storm or a manifestation of profound immunosuppression [28-30]. There is, nevertheless, clear evidence of increased systemic inflammatory markers, including interleukin (IL)-6, IL-8 and IL-1 $\beta$, activation of coagulation pathways, with increased markers such as D-dimer, neutrophil recruitment, activation and extracellular trap formation, deficient production in some patients of antiviral defence mediators, such as interferon- $\alpha$ and $-\beta$, autoimmunity and T-cell activation, among multiple other mechanisms [5, 25, 31-34].

In view of the involvement of both the viral load and host inflammatory response in the disease, repurposing and development of new therapies in COVID-19 has focused primarily on anti-viral, immunosuppressive and immunomodulatory treatments [24, 35-38]. Randomised clinical trials have been conducted at an unprecedented rate to generate evidence for specific interventions [39]. During the early stages of the pandemic in particular, empirical use of antiviral and anti-inflammatory therapies was widespread globally in the absence of formal guidelines or randomised trial evidence [40-43]. It is therefore important to have both recommendations in favour of successful interventions but also evidence to avoid certain therapies if their benefit/risk balance is unfavourable [40].

\section{Scope and objectives of the guideline}

The objective of these guidelines is to provide evidence-based recommendations primarily related to the management of hospitalised adults with COVID-19. This guideline does not address in detail the 
management of COVID-19 in the community. In addition, management in children is not addressed. A guideline cannot address the full complexity of a disease; hence all recommendations should be interpreted considering the clinical circumstances and patients’ perceptions, values and preferences.

The evidence for the management of COVID-19 is accumulating at an unprecedented rate, with new trials published every day. The formal literature review and evidence synthesis process of these guidelines, and the lag time to publication, mean that all guidelines will be "out of date" at the point that they are published. Consequently, the present document represents the most recent update of the European Respiratory Society (ERS) guideline on this topic, which is continuously updated as a "living guideline" with rapid literature searches and updated grading and recommendations as new evidence emerges published as rapid guideline updates on specific topics in the ERS journals (figure 1). The first guideline document was published in March 2021 and this present update was completed in March 2022 and published in August 2022; the previous version of this document is available for reference in the supplementary material.

The target audience for this guideline comprises all stakeholders involved in the care of patients with COVID-19 in hospital. This includes specialists in respiratory medicine, infectious diseases, general internal medicine and multiple other medical and surgical specialities in view of the high prevalence of COVID-19; allied health professionals, including, but not limited to, pharmacists, physiotherapists and nurses; regulatory authorities; pharmaceutical companies; policy-makers; and patients and their families.

Table 1 provides a framework to interpret the recommendations made in this document.

\section{Methods}

\section{Guideline development}

This guideline was developed by an ERS COVID-19 task force chaired by J.D. Chalmers (UK) and N. Roche (France) and utilised the GRADE (Grading of Recommendations, Assessment, Development and Evaluations) methodology [44]. The task force included specialists in respiratory medicine, infectious diseases, guideline methodology, an allied health professional and a patient representative. The task force recommendations have been endorsed by the Chinese Thoracic Society (CTS) and three members of CTS participated as full members of the task force panel.

Due to the COVID-19 pandemic, all panel meetings were held online via teleconference and email, with the initial meeting on 26 June 2020 to identify and prioritise the key topics with the most important

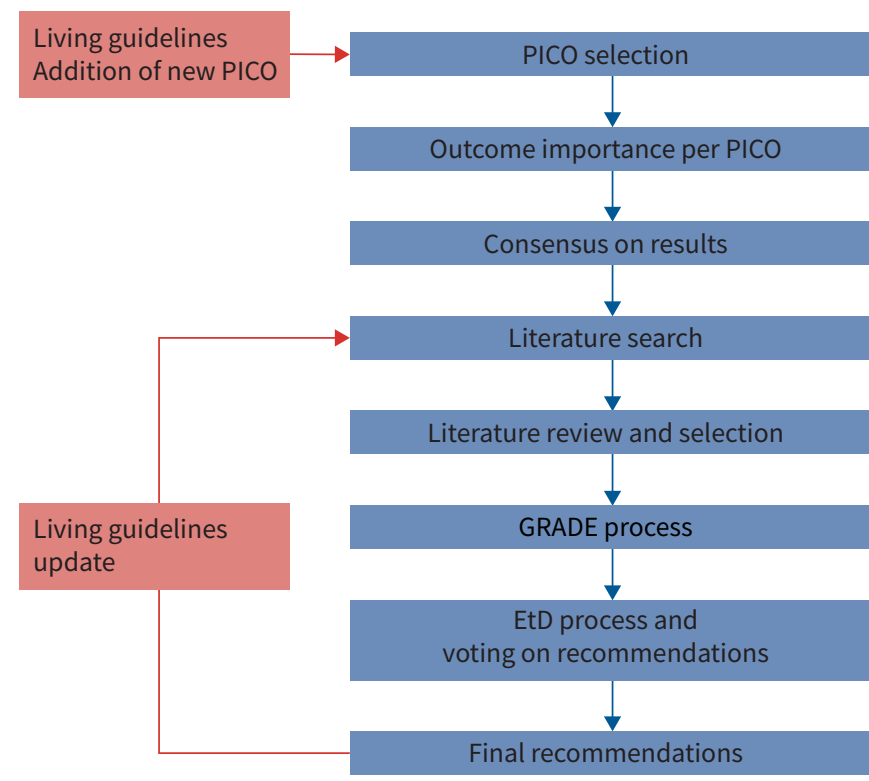

FIGURE 1 Process of guideline development. PICO: Population, Intervention, Comparator, Outcomes; EtD: evidence-to-decision framework. 
TABLE 1 Framework for interpretation of the recommendations made in this document

\begin{tabular}{|c|c|c|}
\hline Target group & Strong recommendations ${ }^{\#}$ & Conditional (weak) recommendations \\
\hline Patients & $\begin{array}{l}\text { All or almost all informed people would choose the } \\
\text { recommended choice for or against an intervention. }\end{array}$ & $\begin{array}{l}\text { Most informed people would choose the recommended course of action, } \\
\text { but a substantial number would not. }\end{array}$ \\
\hline Clinicians & $\begin{array}{l}\text { Most patients should receive the recommended } \\
\text { course of action. }\end{array}$ & $\begin{array}{l}\text { Recognise that different choices will be appropriate for different patients. } \\
\text { Clinicians and other healthcare providers need to devote more time to } \\
\text { the process of shared decision-making by which they ensure that the } \\
\text { informed choice reflects individual values and preferences; decision } \\
\text { aids and shared decision-making are particularly useful. }\end{array}$ \\
\hline Policy-makers & $\begin{array}{l}\text { The recommendation can be adopted as a policy in } \\
\text { most situations. }\end{array}$ & $\begin{array}{l}\text { Policy-making will require substantial debate and involvement of many } \\
\text { stakeholders. }\end{array}$ \\
\hline
\end{tabular}

associated endpoints. From this meeting, the steering group was divided into working groups to focus on specific topics, including anti-virals, anti-inflammatories, anti-coagulants and ventilation strategies.

A total of 11 clinical questions were generated in the first version of the guideline with a further four questions added in the March 2022 update. Questions were formulated using the PICO format (Population, Intervention, Comparator, Outcomes) and systematic reviews were conducted to answer these specific questions. Further details of the literature review process are described below.

\section{Disclosure of potential conflicts of interest}

Committee members disclosed all potential conflicts of interest according to ERS policy. Conflicted members were asked to abstain from discussions and voting on recommendations in which they were considered to have potential conflicts. Compliance with the conflict of interest policy was monitored by the chairs. The methodologists were non-voting members of the panel.

\section{Systematic review}

Two experienced external librarians from KU Leuven libraries (Belgium) designed and ran search strategies using MeSH terms and keywords for each clinical question, in collaboration with the methodology working group (P.C. Goeminne, M.L. Crichton, J.D. Chalmers and T. Tonia). More details of the search strategy are shown in the supplementary material. The search focused on identifying studies that included hospitalised patients with confirmed or highly suspected COVID-19 which included a treatment group and control group that could be used to establish the efficacy and safety of the intervention being studied.

\section{Living guideline updates}

The living guideline format allows for regular updates of the document as new evidence becomes available. The first version of the guideline was published in March 2021. An updated systematic review focusing on mortality was published in December 2021 [46]. The process for updating the living guideline followed a published process for updating systematic reviews used by the World Health Organization (WHO) to update its living guidelines $[47,48]$. The panel used the following framework to determine whether existing PICO questions should be updated:

1) Does the published recommendation still address a current topic for clinical practice or programmes?

2) Are there any new studies or new information relating to the recommendation question?

3) Will any new studies, information or data substantively change the evidence base for the recommendation or the recommendation's credibility?

The guideline panel reviewed each PICO question at a teleconference. Where PICO questions were determined to no longer address a clinically important questions (point 1), where no new studies or new information were available (point 2) and/or there was no realistic prospect that new studies or information would substantively change the evidence base for the recommendation or the recommendations credibility (point 3), the panel determined not to update the literature review and recommendation [47]. Whether new studies or new information was available was based on the expertise and knowledge of the panel in addition to non-systemic searches of the relevant literature. New PICO questions were selected following extensive panel discussions. Previous versions of this guideline document are available for reference as supplementary files to this article. 
Assessment of the level of evidence and degree of recommendations

The panel selected outcomes of interest for each clinical question a priori, based on their relative importance to adult patients with COVID-19 and to clinical decision-making (supplementary material). The importance of outcomes was rated on a nine-point scale (ranging from "not important" to "critical") and only outcomes rated as important or critical for clinical decision-making were included in the evidence tables. We followed the GRADE approach to assess the confidence in the evidence (quality) and the degree of recommendations. The GRADE methodology was used to rate the body of evidence at the outcome level rather than the study level, with assessment of risk of bias at study level performed as described [49].

Recommendations are reported as strong or conditional after considering the quality of the evidence, the balance of desirable and undesirable consequences of compared management options, the assumptions about the relative importance of outcomes, the implications for resource use, and the acceptability and feasibility of implementation. The quality of evidence was rated on four levels (high, moderate, low or very low) based on the GRADE methodology [45]. The overall quality of evidence is then rated as the lowest of the critical outcomes, except where the evidence for all of the critical outcomes favours the same alternative and where the quality of evidence for outcomes that are considered key to clinical decisions takes precedence [50]. Evidence summary of findings tables and evidence-to-decision frameworks were generated for each clinical question (supplementary material). Based on these formats, the panel formulated the clinical recommendations and decided on their strength by consensus, or, if required, by voting. Following the GRADE approach, strong recommendations are worded as "the panel recommends", while conditional recommendations are worded as "the panel suggests" [51].

\section{Guideline}

Table 2 summarises the 22 formal, graded recommendations made within the guideline. In each of the following sections we include a discussion of the underlying evidence and the rationale for the recommendations made. Further details are provided in the evidence tables and evidence-to-decision frameworks provided in the supplementary material.

\section{Recommendations}

We present a total of 22 recommendations based on 15 PICO questions. The recommendations below address a series of therapeutic questions. These recommendations should be used in addition to the basic management of patients with COVID-19 which includes confirmation of the diagnosis using RT-PCR, radiology to identify respiratory complications and supportive care including symptomatic management, fluid management, nutrition, oxygen for patients with respiratory failure, and other aspects of routine care.

PICO 1: In patients hospitalised with COVID-19, should systemic corticosteroids be used compared to usual care?

Recommendations

The panel recommends to offer treatment with corticosteroids to patients with COVID-19 requiring oxygen, non-invasive ventilation or invasive mechanical ventilation (strong recommendation, moderate quality of evidence).

The panel recommends NOT to offer corticosteroids to patients with COVID-19 requiring hospitalisation but not requiring supplementary oxygen or ventilatory support (strong recommendation against, moderate quality of evidence).

Update 2022

We initially reviewed data for six randomised trials meeting the inclusion criteria and a published meta-analysis [37, 52-57]. An updated meta-analysis was performed and published in December 2021 with the addition of two randomised controlled trials [46, 58, 59]. The February 2022 update of this guideline did not include a further analysis on corticosteroids as there was no published data since the first guideline that would materially change the evidence base or the appropriateness of the current recommendation. The meta-analysis shows an odds ratio for mortality of 0.80 (95\% CI 0.64-1.01) from the nine studies.

\section{Summary of evidence}

It is clear that excessive inflammation and a dysregulated immune response play an important role in the progression of severe COVID-19, and therefore there is a strong scientific rationale for the use of anti-inflammatory treatments, particularly in patients with the most severe disease [26, 27, 60, 61]. The largest body of evidence in support of the use of corticosteroids comes from the UK RECOVERY trial, which randomised 2104 patients to dexamethasone $6 \mathrm{mg}$ daily and 4321 patients to standard care in a pragmatic, non-blinded controlled trial [53]. The results demonstrated a statistically significant reduction in 
TABLE 2 Summary of recommendations made in this guideline

\begin{tabular}{l} 
Therapy \\
\hline Corticosteroids \\
IL-6 receptor antagonist \\
monoclonal antibody
\end{tabular}

Hydroxychloroquine

\section{Azithromycin}

\section{Azithromycin and hydroxychloroquine Colchicine}

Lopinavir-ritonavir

Remdesivir

Interferon- $\boldsymbol{\beta}$

\section{Anticoagulation}

Continuous positive airway pressure (CPAP)

High flow nasal oxygen (HFNO)

\section{Convalescent plasma}

Specific anti-SARS-CoV-2 monoclonal antibodies

IL-1 receptor antagonist monoclonal antibody

Recommendation strength

Quality of evidence

1) The panel recommends to offer treatment with corticosteroids for patients with COVID-19 requiring oxygen, non-invasive ventilation or invasive mechanical ventilation

2) The panel recommends NOT to offer treatment with corticosteroids for patients with COVID-19 requiring hospitalisation but not requiring supplementary oxygen or ventilatory support

3) The panel recommends to offer IL-6 receptor antagonist monoclonal antibody therapy to hospitalised patients with COVID-19 requiring oxygen or non-invasive ventilatory support in addition to systemic corticosteroids

4) The panel suggests NOT to offer IL-6 receptor antagonist monoclonal antibody to patients not requiring supplementary oxygen

5) The panel recommends NOT to offer hydroxychloroquine to patients with COVID-19, including hospitalised patients and outpatients

6) The panel recommends NOT to offer azithromycin to hospitalised patients with COVID-19 in the absence of bacterial infection, including hospitalised patients and outpatients

7) The panel recommends NOT to offer hydroxychloroquine and azithromycin in combination to patients with COVID-19

8) The panel recommends NOT to offer colchicine to hospitalised patients with COVID-19

9) The panel recommends NOT to offer lopinavir-ritonavir to hospitalised patients with COVID-19

10) No recommendation is made regarding the use of remdesivir in patients hospitalised with COVID-19 and not requiring invasive mechanical ventilation

11) The panel suggests NOT to offer remdesivir to patients hospitalised with COVID-19 infection who require invasive mechanical ventilation

12) The panel suggests NOT to offer interferon- $\beta$ to hospitalised patients with COVID-19

13) The panel recommends to offer a form of anticoagulation to hospitalised patients with COVID-19

14) The panel suggests to offer non-invasive CPAP delivered through either a helmet or a facemask for patients with COVID-19 and hypoxaemic acute respiratory failure without an immediate need for invasive mechanical intubation

15) The panel suggests to offer HFNO therapy for patients with COVID-19 and hypoxaemic acute respiratory failure without an immediate need for invasive mechanical intubation, and who are unsuitable for CPAP due to intolerance or adverse effects

16) The panel recommends NOT to offer convalescent plasma to hospitalised patients with COVID-19 patients

17) The panel suggests to offer a combination of casirivimab and imdevimab to patients hospitalised with COVID-19 who have no detectable SARS-CoV-2 antibodies (seronegative) and infection with a susceptible variant

18) The panel recommends NOT to offer monoclonal antibodies to patients hospitalised with COVID-19 who have detectable SARS-CoV-2 antibodies (seropositive) or where SARS-CoV-2 antibody status is unknown

19) The panel suggests NOT to offer IL-1 receptor antagonist monoclonal antibodies for hospitalised patients with COVID-19
Strong recommendation for Moderate the intervention

Strong recommendation

Moderate

against the intervention

Strong recommendation for High the intervention

Conditional recommendation against the intervention

Strong recommendation against the intervention

Moderate

Strong recommendation against the intervention

Moderate

Strong recommendation

Moderate gainst the intervention Strong recommendation against the intervention Strong recommendation against the intervention

No recommendation

Moderate

Low

Moderate

Conditional recommendation Moderate against the intervention

Conditional recommendation Very low against the intervention

Strong recommendation for Moderate the intervention

Conditional recommendation High for the intervention

Conditional recommendation Moderate for the intervention

Strong recommendation Low against the intervention

Conditional recommendation Moderate for the intervention

Strong recommendation

Moderate against the intervention

Conditional recommendation Moderate against the intervention 
TABLE 2 Continued

Therapy

20) The panel recommends to offer treatment with baricitinib to hospitalised patients with COVID-19 requiring oxygen, or on high flow oxygen devices, or on non-invasive ventilation

21) The panel suggests NOT to offer JAK inhibitors other than baricitinib to hospitalised patients with COVID-19, except in clinical trials or when other recommended immunomodulators are not available

22) The panel suggests NOT to offer JAK inhibitors to patients with COVID-19 requiring hospitalisation but not requiring supplementary oxygen

\begin{tabular}{|c|c|}
\hline $\begin{array}{l}\text { Strong recommendation for } \\
\text { the intervention }\end{array}$ & High \\
\hline $\begin{array}{l}\text { Conditional recommendation } \\
\text { against the intervention }\end{array}$ & Low \\
\hline $\begin{array}{l}\text { Conditional recommendation } \\
\text { against the intervention }\end{array}$ & Low \\
\hline
\end{tabular}

Grey rows indicate recommendations that are unchanged in the latest update to the guidelines. Orange rows indicate new evidence and/or updates to recommendations in the latest update. IL: interleukin.

mortality with corticosteroid treatment in patients receiving invasive mechanical ventilation at randomisation (41.4\% versus $29.3 \%$ in standard care versus dexamethasone respectively) and a lesser but still statistically significant mortality benefit in those requiring supplementary oxygen at randomisation (26.2\% versus $23.3 \%$ in standard care and dexamethasone respectively) [53]. There was no mortality benefit evident in patients that did not require supplementary oxygen $(14.0 \%$ versus $17.8 \%$ in standard care and dexamethasone respectively) [53]. A systematic review and meta-analysis of critically ill patients with COVID-19, which included data from seven trials, confirms the benefit of corticosteroids on mortality in this population and included data for hydrocortisone and methylprednisolone, suggesting a class effect of steroids (OR 0.70, 95\% CI 0.48-1.01; $\mathrm{p}=0.053$ ) in random effects meta-analysis [54].

The review of the data identified limited evidence on adverse events, and in particular the RECOVERY trial did not report detailed information on safety of the intervention [53]. Data from four trials did not show a significant increase in adverse events (OR 1.09, 95\% CI 0.37-3.18) [37, 52, 55, 56]. Nevertheless, the adverse event profile of corticosteroids is well known, including an increased risk of secondary infections, and should therefore only be used in the appropriate population. These trials have not identified major safety signals to date. Evidence was rated as moderate or high quality for all of the outcomes except for adverse events.

\section{Justification of the recommendation}

The overall risk versus benefit for corticosteroids is favourable. Corticosteroids have been shown to significantly reduce mortality in a large-scale randomised trial and the consistency of results from other trials is reassuring that these data are generalisable. Results were significantly different between subgroups based on the requirement for oxygen, or requirement for mechanical ventilation, with clear absence of benefit in patients not requiring oxygen, justifying different recommendations for different subgroups of patients.

\section{Research recommendations}

Dexamethasone $6 \mathrm{mg}$ daily for 10 days was the regimen selected for RECOVERY and is therefore the regimen that is used as standard [53]. Unanswered questions regarding corticosteroids include the optimal molecule, the optimal timing, dose and scheme, as well as the optimal duration of treatment, long term side-effects and whether other subgroups of patients, such as those not requiring oxygen but with evidence of increased systemic inflammation or radiographic changes, would benefit.

At the time of writing some trials comparing higher dose regimens versus lower dose corticosteroid regimens have been published [62]. The question of corticosteroid dosing will therefore be addressed in a future update of the guideline. We recommend further trials of high versus low dose corticosteroid treatment in COVID-19.

\section{PICO 2: In patients hospitalised with COVID-19, should IL-6 receptor antagonist monoclonal} antibodies be used compared to usual care?

Recommendation

The panel recommends to offer IL-6 receptor antagonist monoclonal antibody therapy to hospitalised patients with COVID-19 requiring oxygen or non-invasive ventilatory support in addition to systemic corticosteroids (strong recommendation, high quality of evidence). 
The panel suggests NOT to offer IL-6 receptor antagonist monoclonal antibody to patients not requiring supplementary oxygen (conditional recommendation against, low quality of evidence).

Update 2022

During the preparation of the first version of the living guidelines, the panel assessed eight randomised, controlled studies comparing the IL-6 receptor antagonist monoclonal antibodies tocilizumab or sarilumab in one or two doses, to usual care [63-70]. Since the publication of the previous version, four additional studies became available, comparing tocilizumab or sarilumab to usual care [71-74].

\section{Summary of evidence}

Observational studies in severe COVID-19 found elevated serum levels of IL-6 that were associated with increased mortality [26, 31, 75]. Several uncontrolled trials suggested a benefit to treatment with IL-6 receptor antagonist monoclonal antibody, with improvement in disease severity and recovery of inflammatory markers [76]. In this update of the living guidelines, the panel assessed a total of 12 randomised controlled trials, comparing IL-6 receptor antagonist monoclonal antibodies (5188 patients in total) to usual care (3615 patients in total) [63-74]. The pooled analysis demonstrated a numerical reduction in mortality which did not achieve statistical significance (1310/5188 (25.3\%) with IL-6 receptor antagonist monoclonal antibodies versus 1068/3615 (29.5\%) with usual care; OR 0.89, 95\% CI 0.781.01). However, the two largest studies, RECOVERY and REMAP-CAP demonstrated a reduction in mortality [68, 69]. Furthermore, there was a significant reduction in the requirement for mechanical ventilation with the use of IL-6 receptor antagonist monoclonal antibodies in seven studies including 4878 patients (14.4\% with IL-6 receptor antagonist monoclonal antibodies versus $18.4 \%$ with usual care; OR $0.75,0.64-0.87)$. Other studies demonstrated an advantage to IL-6 receptor antagonist monoclonal antibodies on composite endpoints of a reduction in combined mechanical ventilation or death (five studies including 4432 patients; OR 0.76, 95\% CI 0.65-0.88). Other key endpoints also support the efficacy of IL-6 receptor antagonist monoclonal antibodies. There were no significant differences regarding adverse events and serious adverse events, and in some of the trials use of IL-6 receptor antagonist monoclonal antibodies was associated with a reduction in infectious complications (possibly due to a reduced need for intensive care unit (ICU) admission) [64, 70].

\section{Rationale}

The findings of reductions in ICU admission, need for mechanical ventilation and composite of mortality or mechanical ventilation were graded as high or critical importance by panel members. The positive outcomes including a reduction in mortality were confirmed in a meta-analysis by the WHO Rapid Evidence Appraisal for COVID-19 Therapies (REACT) Working Group [77]. There were no major safety concerns with the use of IL-6 receptor antagonist monoclonal antibodies.

The trials demonstrated a benefit when IL-6 receptor antagonist monoclonal antibodies were used concomitantly with glucocorticoids. The RECOVERY study performed a pre-specified subgroup analysis regarding the concomitant use of glucocorticoids; the benefit in terms of reduction in mortality was seen only in patients treated with glucocorticoids. Furthermore, some studies which did not demonstrate a benefit to IL-6 receptor antagonist monoclonal antibodies have either excluded patients receiving glucocorticoids [65], or only a minority of participants received glucocorticoids [63, 67, 72].

\section{Research recommendations}

There is a need to clarify the relative benefits of IL-6 receptor antagonist monoclonal antibodies compared to Janus kinase (JAK)2 inhibitors and other potential anti-inflammatory drugs given in combination with corticosteroids. The available data suggests a significant benefit in patients requiring supplementary oxygen and receiving corticosteroids, and there is insufficient data in patients with less severe disease.

\section{PICO 3: In patients hospitalised with COVID-19, should hydroxychloroquine be used compared to usual care? \\ Recommendation}

The panel recommends NOT to offer hydroxychloroquine to patients with COVID-19, including hospitalised patients and outpatients (strong recommendation against, moderate quality of evidence).

Update 2022

Our initial evidence review included 11 randomised studies [36, 78-87]. Following the results of this analysis, the panel judged the question of whether to use hydroxychloroquine as no longer clinically relevant, and so no further updates would be made. In support of this an updated meta-analysis was performed and published in December 2021 analysing an additional six randomised controlled trials [88-93]. 
This shows an updated odds ratio for mortality of 1.09 (95\% CI 0.97-1.22) from 13 trials. Based on these findings, which are consistent with the data reported in the original guideline, the recommendation against hydroxychloroquine remains valid.

\section{Summary of evidence}

Chloroquine and hydroxychloroquine are 4-aminoquinoline drugs primarily used for the treatment of malaria. These agents have immunomodulatory properties and also have in vitro activity against a variety of viruses, including SARS-CoV-2 [94]. Early observational studies of these repurposed medications (alone or in combination with azithromycin) have given divergent results in patients with mild to severe COVID-19 [40]. Despite the preliminary nature of these studies, the reported results have led to confusion about the usefulness of this treatment and its widespread empirical use in some parts of the world [43]. Large randomised controlled studies have now been performed allowing robust analysis of key outcomes in groups of patients with COVID-19 of diverse severity. The results were heavily influenced by the two largest studies performed by the UK RECOVERY group and WHO SOLIDARITY trial [36, 82]. In RECOVERY, participants who received hydroxychloroquine did not have a lower incidence of death at 28 days than those who received usual care [82]. This is in agreement with the interim results of the WHO SOLIDARITY trial, showing no apparent effect of hydroxychloroquine on mortality, irrespective of disease severity at study entry [36]. The pooled estimate for mortality described above effectively excluded a meaningful survival benefit. Besides the absence of a survival benefit, currently available evidence does not show significant positive trends in terms of clinical outcomes, including time to clinical improvements, clinical resolution, deterioration, hospitalisation, ICU admission, and non-invasive or invasive ventilation. Regarding safety, there is an increased risk of adverse events with hydroxychloroquine, such as gastro-intestinal, ocular, liver and cardiac toxicity. Our pooled estimate for adverse effects was OR 4.23 (95\% CI 3.30-5.42), indicating a substantial increase in adverse effects in participants receiving hydroxychloroquine compared to those randomised to the control. Among Brazilian patients hospitalised with mild-to-moderate COVID-19, prolongation of the QT interval was more frequent in patients receiving hydroxychloroquine (alone or with azithromycin), than in those who were not receiving these drugs [78]. In the RECOVERY study, there was a small absolute excess of cardiac mortality of 0.4 percentage points in the hydroxychloroquine group on the basis of very few events [82].

\section{Justification of the recommendation}

There is no evidence of significant clinical benefits associated with hydroxychloroquine, as compared to standard of care, while there is an increased risk of adverse events. Where there is no benefit and evidence of potential harm, a strong recommendation against the intervention is justified.

\section{Future research}

The panel considers that a sufficient number of studies have been performed to conclusively recommend not using hydroxychloroquine in COVID-19 patients. Future studies on this repurposed agent should not be encouraged. The committee recommends studying other antiviral options in well-designed studies of repurposed or SARS-CoV-2 specific medications.

PICO 4: In patients hospitalised with COVID-19, should azithromycin be used compared to usual care? Recommendation

The panel recommends NOT to offer azithromycin to hospitalised patients with COVID-19 in the absence of bacterial infection (strong recommendation against, moderate quality of evidence).

Update 2022

Our literature review identified five randomised trials [78, 95-98]. Two of these are new to this updated analysis [97, 98]. The summary of evidence showed no meaningful effects of azithromycin on mortality, clinical recovery, requirement for mechanical ventilation or length of hospital stay.

\section{Summary of evidence}

Azithromycin has been proposed as a treatment for COVID-19 on the basis of its immunomodulatory actions. There is a need for additional anti-inflammatory/immunomodulatory treatments for hospitalised patients with COVID-19. Evidence suggests that azithromycin has anti-inflammatory effects, which led some to use it for treatment of SARS-CoV-2 earlier in the pandemic.

The largest study was the RECOVERY trial. Between 7 April and 27 November, 2020, of 16442 patients enrolled in the RECOVERY trial, 9433 (57\%) were eligible and 7763 were included in the assessment of azithromycin. In patients admitted to hospital with COVID-19, azithromycin did not improve survival or other prespecified clinical outcomes, such as duration of hospital stay, or the proportion of patients 
discharged from hospital alive within 28 days. Among those not on invasive mechanical ventilation at baseline, no significant difference was seen in the proportion meeting the composite endpoint of invasive mechanical ventilation or death (risk ratio 0.95 , 95\% CI 0.87-1.03; $\mathrm{p}=0.24$ ) [97].

\section{Justification of the recommendation}

No clinical benefits have been clearly demonstrated for use of azithromycin as an anti-inflammatory drug for COVID-19. It is acknowledged that the prevalence of secondary bacterial infection in COVID-19 is not fully established, and that azithromycin may be used for its antibacterial effect in this context. Antimicrobial resistance may result from widespread use of azithromycin if used unnecessarily.

Future research

It is not believed that any further studies of azithromycin for the treatment of COVID-19 are required or will change the recommendations.

PICO 5: In patients hospitalised with COVID-19, should hydroxychloroquine and azithromycin be used in combination compared to usual care?

Recommendation

The panel recommends NOT to offer hydroxychloroquine and azithromycin in combination for hospitalised patients with COVID-19 (strong recommendation against, moderate quality of evidence).

\section{Update 2022}

The initial guideline analysed evidence from one trial investigating the combination of hydroxychloroquine and azithromycin [78], which was later updated in a meta-analysis published in December 2021 to include one further randomised controlled trial [99]. The panel judged the question of whether combined use of hydroxychloroquine and azithromycin should be further analysed as no longer clinically relevant and so no updated searches or meta-analysis was performed.

\section{Summary of evidence}

The potential antiviral and anti-inflammatory effects of azithromycin and hydroxychloroquine are discussed in separate sections above. The use of azithromycin in combination with hydroxychloroquine has been tested in a Brazilian multicentre, randomised, open-label, controlled trial, involving hospitalised patients who were receiving a maximum of $4 \mathrm{~L} \cdot \mathrm{min}^{-1}$ supplemental oxygen [78]. The use of hydroxychloroquine with azithromycin in this population did not improve clinical status at 15 days, as compared with standard care. There was an increased number of adverse events in patients receiving hydroxychloroquine plus azithromycin $(39.3 \%)$ or hydroxychloroquine alone $(33.7 \%)$ than in those receiving none of the trial drugs (22.6\%) [78]. The ProPAC randomised trial was a placebo-controlled trial of hydroxychloroquine and azithromycin administered for 15 days versus placebo. The study was stopped for futility after 117 patients had been randomised with no differences in any clinical outcomes evident between the groups [99].

\section{Justification of the recommendation}

No clinical benefits were noted in two randomised trials where azithromycin was combined with hydroxychloroquine. The panel notes that both azithromycin and hydroxychloroquine have shown no benefits individually and the rationale for the combination providing additional benefits is lacking. The absence of any clinically relevant benefits of hydroxychloroquine or azithromycin alone or in combination supports a recommendation against their use.

Future research

Despite less data for combination therapy compared to the individual drugs, the lack of benefit of hydroxychloroquine or azithromycin alone suggests no further trials of a combination treatment are justified, particularly in light of potential serious cardiac adverse events and other side-effects [100]. The committee recommends studying other antiviral options in well-designed studies of repurposed or SARS-CoV-2-specific medications.

PICO 6: In patients hospitalised with COVID-19, should colchicine be used compared to usual care? Recommendation

The panel recommends NOT to offer colchicine to patients hospitalised with COVID-19 (strong recommendation against, moderate quality of evidence). 
Update 2022

Our literature review identified three randomised controlled trials [101-103], one of which was added during the update in February 2022 [103].

\section{Summary of evidence}

Colchicine is considered to have anti-inflammatory properties through targeting IL-1 and IL-6 in hyperinflammatory syndromes and blocking the inflammasome as well as having in vitro evidence for blocking the coagulation pathway and thrombosis [104-106]. One case-control analysis in COVID-19 suggested survival benefit in patients treated with colchicine as compared to standard of care $(84.2 \%$ versus 63.6\%) [107]. By far the largest study was the RECOVERY trial, which randomised 5610 patients to colchicine (1 mg after randomisation followed by $500 \mu \mathrm{g} 12 \mathrm{~h}$ later and then every $12 \mathrm{~h}$ for 10 days or until discharge) and 5730 to usual care. There was no effect on mortality (rate ratio 1.01, 95\% CI 0.92 1.10) and no effect on other outcomes, including time to discharge and requirement for ICU admission. Two smaller randomised controlled trials were identified in the literature search showing beneficial effects of colchicine. Both studies were assessed as being high risk of bias [101, 102].

A significant increase in adverse events (mainly diarrhoea) was noted with the administration of colchicine (OR 3.70, 95\% CI 1.68-8.16), which may be expected based on longstanding experience with this drug.

\section{Justification of the recommendation}

The largest trial conducted to date comprehensively excludes a potential benefit in hospitalised patients. This lack of clear benefits with an increase in adverse events results in a recommendation against use.

\section{Research recommendations}

At the time of writing a further trial has been published of 1279 patients from Argentina, which was not included in our literature search. This trial also showed no effect of colchicine compared to standard care on mortality, ICU admission or other outcomes, with an increase in diarrhoea [108]. Based on these confirmatory findings we do not recommend further trials into colchicine.

PICO 7: In patients hospitalised with COVID-19, should lopinavir-ritonavir be used compared to usual care?

Recommendation

The panel recommends NOT to offer lopinavir-ritonavir to hospitalised patients with COVID-19 (strong recommendation against, low quality of evidence).

Update 2022

Our evidence review included three randomised trials, including the aforementioned RECOVERY [109] and SOLIDARITY [36] platform trials, plus a Chinese trial by CAO et al. [110]. The guideline panel judged the question of whether to use lopinavir-ritonavir as no longer a relevant clinical question and so no further update would be made. This is supported by an updated meta-analysis focused on mortality published in December 2021 where the odds ratio for mortality was 1.02 (95\% CI 0.91-1.15) from four trials [46, 88]. Based on these findings, which are consistent with the data reported in the original guideline, the recommendation against use of lopinavir-ritonavir remains valid.

\section{Summary of evidence}

Lopinavir is a human immunodeficiency virus (HIV) type 1 aspartate protease inhibitor, which is usually combined with ritonavir to increase its plasma half-life through inhibition of cytochrome P450 [111]. These drugs are widely available as a drug in clinical use for HIV. The combination was shown to reduce the risk of adverse clinical outcomes and viral load among patients with SARS as compared to historical controls [112]. No effect on mortality or other clinical benefits were evident on endpoints including time to clinical improvement, viral load, viral clearance, discharge from hospital within 28 days and invasive mechanical ventilation. Adverse events and serious adverse events were not increased.

\section{Justification of the recommendation}

Lopinavir-ritonavir has a known adverse event profile and significant drug-drug interactions which present potential for patient harm [113, 114]. Therefore, clear evidence of efficacy would be required to recommend its use. The literature review found no evidence of benefit across three randomised controlled trials. As the drug is not effective and may theoretically be harmful, this justifies a strong recommendation against its use even considering the low quality of available evidence. 
Research recommendations

As several trials show no benefit, no further trials of lopinavir-ritonavir in this population are justified.

PICO 8: In patients hospitalised with COVID-19, should remdesivir be used compared to usual care? Recommendation

The panel makes no recommendation regarding the use of remdesivir in patients hospitalised with COVID-19 and not requiring invasive mechanical ventilation (no recommendation, moderate quality of evidence).

The panel suggests NOT to offer remdesivir to patients hospitalised with COVID-19 who require invasive mechanical ventilation (conditional recommendation against, moderate quality of evidence).

Update 2022

The panel discussed this recommendation in September 2021 and determined that, while the question of whether or not to use remdesivir remains relevant, no new data had been published that would alter the validity of the recommendation. The recommendation described above remains valid based on five randomised controlled trials [36, 115-118], with one additional trial included in the meta-analysis focused on mortality [46].

\section{Summary of evidence}

Remdesivir is an inhibitor of the viral RNA-dependent RNA polymerase. It has proven effective in vitro against SARS-CoV-1, MERS-CoV and SARS-CoV-2 $[119,120]$. A reduction in time to recovery and length of hospital stay was demonstrated for remdesivir in one trial (ACTT1) [118]. This trial randomised 1062 patients (541 to remdesivir and 521 to placebo) [118]. The primary outcome of recovery time was reduced from 15 days to 10 days (rate ratio for recovery 1.29, 95\% CI 1.12-1.48; $\mathrm{p}<0.001$ ). Length of hospital stay was also reduced from a median of 17 days to 12 days, and other secondary endpoints showed positive benefits [118]. In contrast, no clinical benefits were demonstrated in the other trials, including the large SOLIDARITY trial, which found no evidence of a mortality benefit. The SOLIDARITY analysis of remdesivir included 2743 patients receiving active treatment and 2708 controls. Mortality was not impacted, with a rate ratio of 0.95 (95\% CI 0.81-1.11; p=0.50) [36]. The SOLIDARITY group also included an updated meta-analysis of existing trials including ACTT1, SOLIDARITY and additional trials that randomised patients 2:1, and concluded there was no mortality benefit of remdesivir (rate ratio $0.91,95 \%$ CI 0.79-1.05) [36]. Our review identified very similar results with an odds ratio for mortality of 0.92 (95\% CI 0.79-1.07), with no increase in adverse events (OR 1.05, 95\% CI 0.71-1.55) from three studies.

In ACTT1, no benefit on the primary outcome of clinical recovery (recovery rate ratio 0.98 , 95\% CI 0.70 1.36) was observed in patients who started remdesivir when they were already on mechanical ventilation or extracorporeal membrane oxygenation [118].

Justification of the recommendation

The panel considers that time to recovery and length of hospital stay are relevant clinical endpoints in the absence of a mortality benefit of remdesivir. Nevertheless, these benefits have been demonstrated in only one randomised trial. The reported benefits are regarded by the panel as modest. The lack of significant adverse effects means that the balance of benefit versus risk was considered marginally in favour of the intervention by some members of the panel but not by others. The panel discussed this topic extensively and voted on the final recommendation, resulting in no majority favouring either a recommendation for or a recommendation against remdesivir use. The panel therefore makes no recommendation regarding remdesivir in patients not requiring invasive mechanical ventilation. In GRADE methodology this is referred to as a conditional recommendation for the intervention OR the alternative. This recommendation does not indicate that clinicians should use remdesivir routinely or that clinicians should avoid use of remdesivir in all cases. Rather it indicates that the balance of risks and benefits is uncertain and its use by patients should ideally be in the context of a randomised clinical study, or where patients have been fully informed of the risks and benefits. If treatment is given it should be given for 5 days based on evidence that this is at least as effective as 10 days administration [121]. Liver function tests should be checked prior to administration of remdesivir and checked while patients are on treatment; remdesivir should not be prescribed in patients with severe renal dysfunction (glomerular filtration rate $<30 \mathrm{~mL} \cdot \mathrm{min}^{-1}$ ).

Subgroup effects were observed with no benefit on the primary outcome evident in patients requiring invasive mechanical ventilation or extracorporeal membrane oxygenation. As this outcome is the main benefit supporting any use of remdesivir, the panel considers it appropriate to make a subgroup 
recommendation against remdesivir use in these patients where clear absence of benefit has been demonstrated. Availability and cost are important considerations for some healthcare systems.

Future research

As the benefit is unclear, further large studies in hospitalised patients, including endpoints such as clinical improvement, clinical deterioration and length of stay, should be performed to confirm the results of ACTT1. Identifying subgroups of patients who benefit is a priority, based on timing of administration and requirement for oxygen. The benefit of remdesivir in addition to systemic corticosteroids and other anti-inflammatory agents, which are now regarded as standard of care for COVID-19, needs to be established. We highlighted in the first version of the guidelines that there are strong theoretical reasons to believe anti-viral treatments will be more effective when given earlier in the disease course and this appears to have been demonstrated by a study showing that remdesivir given early in the disease in the community reduced the risk of hospitalisation [122].

PICO 9: In patients hospitalised with COVID-19, should interferon- $\beta$ be used compared to usual care? Recommendation

The panel suggests NOT to offer interferon- $\beta$ to hospitalised patients with COVID-19 (conditional recommendation against, very low quality of evidence).

Update 2022

The panel determined that whether or not to use systemic interferon- $\beta$ treatment was not a priority clinical question at a meeting in September 2021, and that no further data had been published to suggest the existing recommendation should be changed. As such the current recommendation against the treatment remains valid based on four randomised controlled trials [36, 123-125] with one additional trial included in the meta-analysis focused on mortality [46].

\section{Summary of evidence}

Interferons are signalling proteins released by host cells as a component of innate immune system in response to viral infections [6, 126]. Type 1 interferons have in vitro activity against coronaviruses [127], and in vivo promoted improved symptoms and viral clearance as part of a triple therapy regimen also containing lopinavir-ritonavir and ribavirin compared to lopinavir-ritonavir alone [128]. There is evidence that SARS-CoV-2 suppresses innate interferon release and the extent of this is linked to disease severity [126]. All of this provides a sound rationale for evaluating interferon as a therapy for COVID-19.

Two small proof-of-concept trials showed large benefits, including reduced mortality [123, 124], but a much larger trial (the WHO SOLIDARITY trial) suggests no evidence of benefit and potential harm (rate ratio 1.16 , $95 \%$ CI $0.96-1.39 ; \mathrm{p}=0.11$ ). Our pooled estimate showed no statistically significant mortality benefit (OR 0.55, 95\% CI 0.18-1.63) or benefit on clinical deterioration. The quality of evidence was rated as very low.

\section{Justification of the recommendation}

Clinical benefit has not been clearly demonstrated for systemic interferon treatment. The largest trial of this drug showed no effect on mortality and a trend towards an increase in mortality. Safety data is incompletely reported across all trials. In the absence of clear benefit or safety, a recommendation for use cannot be made. The conditional recommendation is based on very low quality of evidence.

Future research

A phase 2 trial demonstrated a significant benefit of inhaled interferon- $\beta-1 \mathrm{a}$ in 101 patients conducted in the UK [129]. While small trials should be treated with caution, this suggests the possibility that inhaled delivery has a different effect to systemic delivery of interferon. Further studies to investigate the efficacy of inhaled interferon- $\beta$ are justified.

PICO 10: In patients hospitalised with COVID-19, should anticoagulants be used compared to usual care? Recommendation

The panel recommends to offer a form of anticoagulation to hospitalised patients with COVID-19 (strong recommendation, moderate quality of evidence). 
Update 2022

The literature search identified five randomised controlled trials comparing high dose (therapeutic or intermediate) to lower dose (prophylactic or intermediate) anticoagulation [130-134], which supersedes the narrative analysis of observational studies presented in the previous version of these guidelines.

Summary of the evidence

Thromboembolic complications, including pulmonary emboli, are common in patients hospitalised with COVID-19 [135], while vascular dysfunction, including immunothrombosis, contributes to the severity of disease [136, 137]. In patients hospitalised for medical conditions, a high risk of thrombotic complications has long been recognised and prophylactic anticoagulation has been shown to be efficacious, safe and cost-effective. Given these results, a placebo-controlled trial in patients hospitalised for COVID-19 would appear to be unethical. Accordingly, no trial comparing anticoagulation to placebo or to no anticoagulation was identified, but observational studies suggest a mortality benefit for using any form of anticoagulation compared to no anticoagulation.

One study compared rivaroxaban or therapeutic dose low molecular weight heparin (LMWH) or unfractionated heparin to prophylactic dose LMWH or heparin [130]. Two studies used therapeutic LMWH [131, 132]. Two studies allowed either LMWH or unfractionated heparin [133, 134].

In the pooled analysis of the five trials, mortality was 346/2164 (16.0\%) in the intervention groups versus $343 / 2048(16.7 \%)$ in the control groups (risk ratio 1.01, 95\% CI 0.88-1.14), showing no mortality benefit of the intervention. None of the individual trials showed a mortality benefit meeting conventional statistical significance.

There was a significant reduction in major thrombotic events with treatment from $7.2 \%$ in the control groups to $4.0 \%$ in the treatment groups. There was a significance increase in major bleeding events $(3.4 \%$ versus 1.5\%; OR 2.39, 95\% CI 1.56-3.66). Subgroup analyses of outcomes in non-ICU and ICU admitted patients did not identify significant differences in efficacy.

\section{Justification of the recommendation}

All patients hospitalised with COVID-19 should receive a form of anticoagulation, which is considered standard of care for all patients. The guideline panel has not expressed a preference for prophylactic dose or therapeutic dose anticoagulation. This is because the evidence does not provide a compelling argument for either approach. Therapeutic dose anticoagulation reduces the risk of major thrombosis at the expense of increased major bleeding. Whether biomarkers could identify patients at higher risk of thrombotic events or major bleeding should be the focus of future research. No effect of therapeutic anticoagulation on mortality was shown. Of note, patients at highest risk of pulmonary embolism-related death, i.e. those with clinically diagnosed or strongly suspected pulmonary embolism or deep-vein thrombosis, and those with established risk-factors for bleeding, were not included in the trials. As hospitalisation with COVID-19 is a risk for venous thromboembolism, we suggest a low threshold for investigating the possibility of thromboembolism in patients with COVID-19. If anticoagulation is necessary for reasons different from COVID-19 (e.g. atrial fibrillation) it should be provided as recommended in the corresponding guidelines.

\section{Research recommendation}

Larger trials are needed to establish whether therapeutic anticoagulation provides benefits over prophylactic dose anticoagulation and to determine whether biomarkers or risk factor stratification can be used to identify the optimal patient population to receive therapeutic dose anticoagulants.

PICO 11: In patients hospitalised with COVID-19, should continuous positive airway pressure or high flow nasal oxygen, with or without adjunctive strategies such as prone positioning, be used compared to usual care (defined as the absence of these interventions or invasive mechanical ventilation)?

\section{Recommendations}

The panel suggests to offer non-invasive continuous positive airway pressure (CPAP) delivered through either a helmet or a facemask for patients with COVID-19 and hypoxaemic acute respiratory failure (hARF) without an immediate need for invasive mechanical intubation (conditional recommendation, high quality of evidence).

The panel suggests to offer high flow nasal oxygen (HFNO) therapy for patients with COVID-19 and hARF without an immediate need for invasive mechanical intubation and who are unsuitable for CPAP due to intolerance or adverse effects (conditional recommendation, moderate quality of evidence). 
Update 2022

No randomised trials were identified by our literature search in the previous version of the guidelines. Our updated 2022 guidelines include three trials [138-140] which investigated the use of CPAP or HFNO.

Summary of the evidence

This question was addressed using of a limited number of randomised trials, and partly by narrative account [138-140]. Non-invasive respiratory support strategies are helpful if they reduce mortality and morbidity and decrease intubation and ICU admission rates. Prior to the emergence of SARS-CoV-2, a network meta-analysis showed that both non-invasive ventilation and HFNO reduced intubation in hARF, but only helmet and mask non-invasive ventilation reduced mortality. However, only a minority of trials in this meta-analysis evaluated CPAP, and it cannot be assumed these results are applicable to patients with acute respiratory failure due to COVID-19 [141].

At the beginning of the pandemic, there were concerns that non-invasive strategies might delay intubation, and were classified as "aerosol generation procedures". However, the use of non-invasive strategies increased progressively across the pandemic waves following both the impact of other therapies and vaccines that reduced disease severity, and the increased confidence in these techniques [142]. Initial case series studies have now been superseded by randomised trials that predominantly compared non-invasive CPAP and HFNO with standard oxygen therapy (SOT). The largest trial, RECOVERY-RS, showed that CPAP reduced the combined primary endpoint of need for intubation and mortality at 30 days compared to SOT, whereas HFNO showed no benefit compared with SOT [140]. The outcome in the CPAP arm was driven by reduction in intubation. Both mask and helmet CPAP were used, although more patients underwent mask CPAP treatment. While the limitations of this trial were that it was not protocolised, there were significant crossovers between groups, and the intended recruitment number was not reached, a strength was that the study reflected clinical practice and included patients treated on respiratory high dependency units [140].

A second open-label trial randomised patients with COVID-19 pneumonia to SOT or HFNO, showing a reduction in intubation rate and time to recovery in the HFNO group, while length of stay was not affected [138]. In a physiological randomised controlled trial enrolling a small number of patients, TENG et al. [139] showed a faster improvement in oxygenation in patients on HFNO and shorter ICU stay compared those on SOT. The RECOVERY-RS trial showed a slightly higher rate of barotrauma in patients undergoing CPAP compared to HFNO [140]. Ospina-TAscón et al. [138] showed no difference in secondary pneumonia rate between HFNO and SOT.

An open feasibility study showed that delivery of both HFNO and CPAP can be safely carried out in a high dependency unit in patients with single organ failure. Ongoing studies suggest that with the use of modified circuits and personal protective equipment, dissemination of the infection from CPAP and HFNO devices may be low and, ultimately, risks depend on the infectivity of patient and specific manoeuvres [143-145].

Overall results show that CPAP probably offers an advantage over HFNO. It should be noted that randomised controlled trials included patients deemed suitable for intubation and did not recruit patients with a ceiling of non-invasive respiratory support. Thus, conclusions cannot be extended to these groups. Based on the number of crossovers, it is also clear that not all patients could tolerate CPAP therapy and HFNO may be appropriate in these people [140].

Prone positioning of non-intubated patients with hARF due to COVID-19 pneumonia has been recently tested across different settings including emergency departments, hospital wards, or in ICUs as an adjunct to conventional oxygen therapies [146-149]. A large heterogeneity across these experiences can be recognised. They differ in terms of patient selection, type of oxygen therapy support used, setting, timing and duration of the intervention and, therefore, provide variable results. Despite this heterogeneity, reports document a significant improvement in oxygenation and respiratory rate upon prone positioning, and the majority were able to tolerate the procedure.

Justification of the recommendation

Reducing the need for invasive ventilation and pressure on ICU healthcare resources would be highly advantageous. CPAP use reduces requirement for oxygen delivery in situations of low oxygen supply availability.

The application of CPAP and HFNO should not delay intubation and mechanical ventilation in patients who fail to respond to a non-invasive approach. CPAP and HFNO therapies are classified as 
aerosol-generating procedures and should be used with healthcare professionals in full personal protective equipment. The nature of aerosol generation or dispersion when using CPAP and HFNO has been explored using a range of imaging, particle sizing and virus sampling studies, producing mixed results [143-145]. Benefits of CPAP and HFNO should be balanced against risks.

\section{Research recommendations}

Further randomised studies, especially head-to-head comparisons addressing the optimal mode of ventilation in patients with hARF and COVID-19, are required. It would also be helpful to explore outcomes of non-invasive strategies in patients not suitable for ICU admission, and the long term impact on pulmonary function.

PICO 12: In patients hospitalised with COVID-19, should convalescent plasma be used compared to usual care?

Recommendation

The panel recommends NOT to offer convalescent plasma to hospitalised patients with COVID-19 (strong recommendation against, low quality of evidence).

Update 2022

Convalescent plasma was added to the guideline in the most recent update.

\section{Summary of the evidence}

The rationale for convalescent plasma is that passive immunity may aid viral clearance during early infection before the specific immune response of the host is established. It was viewed as a promising candidate therapy for COVID-19 based on encouraging case series in SARS and Middle East respiratory syndrome (MERS) [150]. This was further encouraged by initial observational cohort studies suggesting reduced mortality in users of plasma therapy, and an advantage of high antibody titre over low antibody titre plasma [151]. Based on these data, convalescent plasma received emergency use authorisation in the USA and was used extensively worldwide.

Convalescent plasma is one of the most intensively studied interventions in hospitalised patients with COVID-19. At the completion of our literature review, the available evidence included 13 published papers [152-164] and four preprints [165-168]. Our review and meta-analysis found no beneficial effect of convalescent plasma on mortality. Mortality was 1704/7673 (22.2\%) compared to $1643 / 7154$ patients in the control arms (OR 0.97, 95\% 0.89-1.04). No other clinically relevant benefits were demonstrated and there was also no significant increase in adverse events or serious adverse events in the trials. In addition, none of the 17 individual studies reviewed showed a significant benefit.

\section{Justification of the recommendation}

Convalescent plasma has been extensively studied. While trials differ in their design, duration, endpoints and titre of plasma used, the results are highly consistent and suggest no meaningful clinical benefit with therapy. While no adverse effects were noted in the trials, transfusion of blood products is not without risks, and this justifies a strong recommendation against treatment.

\section{Research recommendations}

None. There is sufficient evidence to conclude no role for convalescent plasma in the management of severe COVID-19.

PICO 13: In patients hospitalised with COVID-19, should specific anti-SARS-CoV-2 monoclonal antibodies be used compared to usual care?

Recommendation

The panel suggests to offer a combination of casirivimab and imdevimab to patients hospitalised with COVID-19 who have no detectable SARS-CoV-2 antibodies (seronegative) and have infection with a susceptible variant (conditional recommendation, moderate quality of evidence).

Since the variant causing infection in an individual patient is often not known, treatment may be considered in regions and at times with a predominance of SARS-CoV-2 variant(s) susceptible to at least one of these monoclonal antibodies. Where resistant variants are dominant, variant identification should be performed to ensure that a susceptible variant is involved before confirming administration. 
The panel recommends NOT to offer monoclonal antibodies to patients hospitalised with COVID-19 who have detectable SARS-CoV-2 antibodies (seropositive) or where SARS-CoV-2 antibody status is unknown (strong recommendation against, moderate quality of evidence).

Update 2022

This therapy was added to the guideline in the most recent update.

\section{Summary of evidence}

The literature search identified two platform trials of monoclonal antibodies against SARS-CoV-2 epitopes in patients hospitalised for COVID-19 eligible for this meta-analysis [169, 170]. One trial from the RECOVERY platform evaluated casirivimab and imdevimab versus usual care in 9785 patients, of whom $32 \%$ were seronegative for SARS-CoV-2. The other (TICO platform) assessed LY-CoV555 versus placebo in 314 patients, the vast majority of whom (95\%) also received remdesivir.

In the RECOVERY study, a pre-specified sub-analysis was conducted in seronegative patients. A significant $18 \%$ reduction in mortality was shown and a trend was observed for a reduced risk of progression to invasive mechanical ventilation following the use of monoclonal antibodies (rate ratio 0.88 , 95\% CI 0.73-1.06). The risk of death or mechanical ventilation (composite outcome) was significantly reduced, by $17 \%$. There was a significant $19 \%$ increase in the rate of discharge alive from the hospital at day 28 (64.8\% versus 58.4\%).

No meaningful treatment responses were seen in other outcomes. No significant benefit was observed in seropositive patients nor in the whole population (seronegative+seropositive). Subgroup analyses did not identify response modulators other than the serological status.

\section{Justification of the recommendation}

In seronegative patients, a beneficial effect in terms of mortality and need for mechanical ventilation has been demonstrated, while no safety issues were identified, resulting in a favourable benefit/risk profile. These data were generated during a period of the pandemic when many patients were unvaccinated, and before the omicron variant became dominant in many countries. The combination of casirivimab and imdevimab is not effective against the omicron variant. Consequently, the recommendation to consider use of this combination is only in cases where the variant is known to be susceptible, or the dominant variant is susceptible.

\section{Research recommendations}

Further research is ongoing to assess other neutralising antibodies or their combinations in patients hospitalised for COVID-19, with different target epitopes and variants. Identifying optimal target populations will be required based on patient, virus and disease characteristics, as well as concomitant therapies and vaccination status.

PICO 14: In patients hospitalised with COVID-19, should anti-IL-1 receptor monoclonal antibodies be used compared to usual care?

Recommendation

The panel suggests NOT to offer IL-1 receptor antagonist monoclonal antibodies for hospitalised patients with COVID-19 (conditional recommendation against, moderate quality of evidence).

Update 2022

This therapy was added to the guideline in the most recent update.

\section{Summary of the evidence}

The literature search identified three published papers [171-173] and one preprint [174] describing randomised controlled trials of IL-1 receptor monoclonal antibodies against standard care or placebo in COVID-19 patients. The evidence summary showed mortality of 183/1052 (17.4\%) in the active treatment group and 192/872 (22.0\%) in the control groups. The pooled effect estimate from the four trials was an odds ratio of 0.97 (95\% CI 0.76-1.24). No significant increase in adverse events was observed. One trial was clearly an outlier from the other trials in the analysis and showed large benefits of active treatment. The SAVE-MORE trial of 594 patients in Greece enriched the patient population using a biomarker, soluble urokinase plasminogen activator receptor in serum (suPAR). In this study large improvements in all clinical outcomes including survival (hazard ratio 0.45, 95\% CI 0.20-0.99) and clinical status were observed [173]. $86 \%$ of patients in the trial were receiving dexamethasone. The REMAP-CAP platform 
trial was the largest trial contributing to our analysis and found no evidence of mortality benefit or any other clinical benefits in a critically ill population [174].

\section{Justification of recommendations}

Although one study found a beneficial effect of IL-1 receptor mononclonal antibody treatment, the number of mortality events was small and the study biomarker strategy requires additional validation [173]. The role of this treatment on top of anti-IL-6 receptor monoclonal antibodies and JAK2 inhibitors is unknown. In view of the larger evidence base for anti-IL-6 and JAK2 inhibitor therapies, these are likely to be preferred, and it is currently unknown if they can be given in combination.

\section{Research recommendation}

Independent validation of the efficacy of anti-IL-1 $\beta$ monoclonal antibody treatment guided by biomarkers is required. Since the expected benefits of anti-IL-6 receptor monoclonal antibodies and JAK2 inhibitors overlap with this therapy, it will be important to determine whether an anti-IL-1 strategy is additive to these other therapies, or if it carries any advantages as an alternative therapy.

PICO 15: In patients hospitalised with COVID-19, should JAK inhibitors be used compared to usual care? Recommendation

The panel recommends to offer treatment with baricitinib to hospitalised patients with COVID-19 requiring oxygen, or on high flow oxygen devices, or non-invasive ventilation (strong recommendation, high quality of evidence).

The panel suggests NOT to offer JAK inhibitors other than baricitinib to hospitalised patients with COVID-19, except in clinical trials or when other recommended immunomodulators are not available (conditional recommendation against, low quality of evidence).

The panel suggests NOT to offer JAK inhibitors to patients with COVID-19 requiring hospitalisation but not requiring supplementary oxygen (conditional recommendation against, low quality of evidence).

\section{Update 2022}

This therapy was added to the guideline in the most recent update.

\section{Summary of evidence}

Janus kinases are a class of intracellular tyrosine kinases mediating downstream signals of types I and II cytokine receptors [175]. Levels of various type I and II cytokines, such as IL-6, IL-10, and tumour necrosis factor- $\alpha$, were reported to be elevated significantly in patients with severe COVID-19 [75]. When these cytokines bind to their receptors on target cells, the JAK/STAT (signal transducer and activator of transcription) signalling pathway would be activated to mediate transcriptional regulation of target genes [175]. Therefore, JAK inhibitors that can block the signal transduction of pro-inflammatory cytokines becomes a potent therapeutic strategy for COVID-19 [176].

Our evidence review included four randomised controlled trials [177-180]. Following the completion of our literature searches an additional trial of baricitinib in critically ill patients was published [181] and the RECOVERY trial results were released as a preprint which included an updated meta-analysis [182]. A proof-of-concept study of an inhaled JAK inhibitor was not pooled with the other studies [183].

The meta-analysis of four trials showed a mortality rate of 90/1443 (6.2\%) in the active treatment groups and 148/1445 (10.2\%) in the control groups. Two studies examined baricitinib, one study used ruxolitinib and one study used tofacitinib [177-180]. The pooled odds ratio for mortality was 0.58 (95\% CI $0.44-$ 0.76) indicating a large survival benefit associated with JAK inhibitor treatment. RECOVERY showed a lower effect estimate for mortality compared to some earlier studies and had a higher overall mortality rate. This is likely to reflect the pragmatic design of RECOVERY allowing the inclusion of patients that may be excluded from more selective randomised controlled trials. Other benefits, including reduced progression to requirement for mechanical ventilation, were observed. JAK inhibitors did not increase the incidence of adverse events (OR 0.94, 95\% CI 0.81-1.09), from four studies and serious adverse events were reduced with JAK inhibitor therapy (OR 0.77, 95\% CI 0.63-0.94). This is in agreement with the results of one recently published living meta-analysis, which included data from thirteen clinical studies (six randomised controlled trials and seven observational studies) and demonstrated a benefit in mortality in JAK inhibitors group (risk ratio 0.62, 95\% CI 0.49-0.78). In the subgroup analysis for randomised controlled trials of different JAK inhibitors, only baricitinib decreased mortality (risk ratio 0.64, 95\% CI 0.51-0.80) [184]. 
Following completion of our systematic review, the RECOVERY trial published data on a further 8156 patients randomised to baricitinib or usual care, demonstrating a significant reduction in mortality (age-adjusted rate ratio $0.87,95 \%$ CI $0.77-0.98$ ) [182]. A meta-analysis incorporating these data supported a $20 \%$ reduction in mortality. Importantly, 95-96\% of patients were receiving corticosteroids and $23 \%$ were receiving tocilizumab in RECOVERY and the efficacy and safety of baricitinib was not affected by co-administration with tocilizumab [182].

\section{Justification of the recommendation}

JAK inhibitors were shown to reduce mortality of hospitalised COVID-19 patients. In terms of safety, there was no increase of adverse events and a lower frequency of serious adverse events with active treatment. However, current evidence mainly supports the clinical benefits of baricitinib. Randomised controlled trials of other JAK inhibitors have relatively small sample sizes ( $n=289$ for tofacitinib, $n=41$ for ruxolitinib and $\mathrm{n}=25$ for nezulcitinib), and none of these trials demonstrated significant mortality benefits.

For optimal patient population, subgroup analysis showed patients benefit more from the baricitinib treatment if they are receiving supplementary oxygen or non-invasive respiratory support. A similar patient population most likely to benefit was observed in analyses of other immunomodulatory drugs, such as corticosteroids and IL-6 receptor antagonist monoclonal antibodies. As sufficient evidence was only available for baricitinib, the panel recommends offering baricitinib to hospitalised patients with COVID-19 requiring oxygen, or on high flow oxygen devices or non-invasive ventilation. It should be noted that less evidence is available regarding the initiation of baricitinib in patients requiring invasive mechanical ventilation, as only 251 patients in RECOVERY were in this category. It is recommended that baricitinib is initiated earlier in the disease course and before the requirement for mechanical ventilation.

Baricitinib should be considered only as an add on therapy to corticosteroids. JAK inhibitors and IL-6 receptor monoclonal antibodies have a similar role in therapy and a related mechanism of action. RECOVERY showed that they can be combined with no reduction in efficacy and safety [182]. Combination therapy has economic and clinical considerations and so may not be appropriate for all patients. Those at highest risk of deterioration, such as those requiring non-invasive ventilation, may be the optimal patient population and indeed this subgroup had the highest efficacy of baricitinib treatment in RECOVERY (mortality reduced from $25 \%$ to $20 \%$; age-adjusted rate ratio 0.75 , 95\% CI $0.62-0.90$ ).

\section{Research recommendations}

Unanswered questions regarding JAK inhibitors include benefits of other JAK inhibitors, such as ruxolitinib, tofacitinib and nezulcitinib, dose and time to start treatment, the frequency and duration of treatment, and the optimal concomitant treatment. In addition, further research is needed to identify the optimal patient population for treatment with JAK inhibitors, especially the role of inflammation biomarkers in identifying responders, and regarding choice of anti-IL-6 antibodies versus JAK inhibitors and when it is optimal to combine anti-inflammatory drugs.

\section{Summary and further considerations}

The guideline recommendations are summarised in figure 2. The overall aim of management of hospitalised patients with COVID-19 is to reduce mortality and prevent complications, including requirement for ICU admission and prolonged length of hospital stay. This guideline indicates that corticosteroids, IL-6 receptor antagonists, monoclonal antibodies (in seronegative patients) and JAK inhibitors are all associated with decreased mortality and improvements in other outcomes. CPAP is recommended as the non-invasive ventilation modality of choice with HFNO therapy recommended in patients intolerant or unsuitable for CPAP. Anticoagulation is recommended for all hosptialised patients. We also identify several new and repurposed therapies that currently do not have evidence to support their use. Despite the availability of these therapies there remains a high burden of disease in hospital confirming the need to identify additional effective therapies.

The recommendations in this guideline are derived from a systematic literature review and standardised GRADE methodology. Our recommendations may therefore differ from other documents and from recommendations by regulatory authorities that use different methodology. The purpose of guidelines is to improve the quality of care that patients receive and to standardise care across different healthcare settings and systems by identifying those therapies which have a high level of evidence supporting their use. This guideline should be used as a starting point for treatment algorithms which have to be modified as additional data accumulates, taking into consideration also local availability of therapies, SARS-CoV-2 epidemiology, including variants, and recommendations from national and international regulatory authorities. 


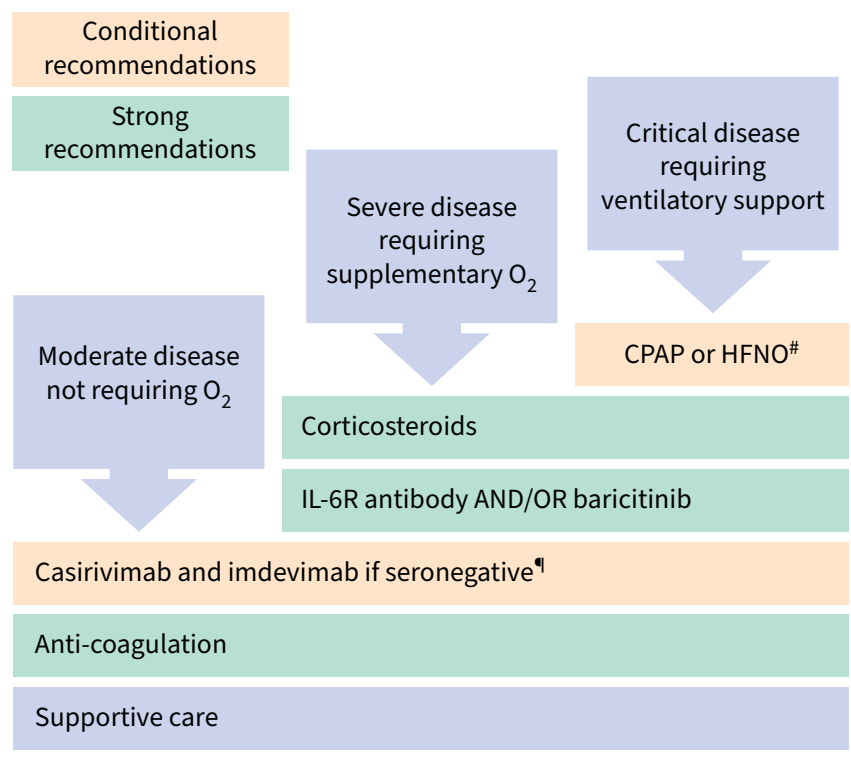

FIGURE 2 Summary of the European Respiratory Society guideline for management of hospitalised patients with COVID-19. ": high flow nasal oxygen (HFNO) is recommended where continuous positive airway pressure (CPAP) is contraindicated or not tolerated. ": in addition, this therapy is only recommended where a susceptible variant is dominant, or rapid testing is available to confirm a susceptible variant. IL: interleukin.

This is a living guideline, with the panel continuously reviewing new evidence as it arises. Recommendations for additional therapies not addressed in this guideline will be added in future versions, along with updates on the therapies already reviewed once new data are available.

Acknowledgements: The authors wish to thank Kristel Paque and Krizia Tuand, the biomedical reference librarians of the KU Leuven Libraries - 2Bergen - Learning Centre Désiré Collen (Leuven, Belgium), for their help in conducting the systematic literature search. This guideline was developed in collaboration with the Chinese Thoracic Society (CTS).

This document was endorsed by the European Respiratory Society (ERS) Executive Committee on 9 June 2022 and by the Chinese Thoracic Society on 19 July 2022. This living guideline was updated and republished in August 2022. The original version is archived as a supplementary file to this article. For details of the changes made in this update, please refer to the editorial published in the August 2022 issue of the European Respiratory Journal: https://doi.org/10.1183/13993003.00803-2022

The guidelines published by the European Respiratory Society (ERS) incorporate data obtained from a comprehensive and systematic literature review of the most recent studies available at the time. Health professionals are encouraged to take the guidelines into account in their clinical practice. However, the recommendations issued by this guideline may not be appropriate for use in all situations. It is the individual responsibility of health professionals to consult other sources of relevant information, to make appropriate and accurate decisions in consideration of each patient's health condition and in consultation with that patient and the patient's caregiver where appropriate and/or necessary, and to verify rules and regulations applicable to drugs and devices at the time of prescription.

Conflict of interest: N. Roche reports grants and personal fees from Boehringer Ingelheim, Novartis and Pfizer, personal fees from GSK, AstraZeneca, Chiesi, Sanofi and Zambon, outside the submitted work. M.L. Crichton reports personal fees from AstraZeneca, outside the submitted work. P.C. Goeminne reports personal fees for lectures from GSK and AstraZeneca, grants, personal fees for advisory board work and non-financial support for meeting attendance from Chiesi, outside the submitted work. B. Cao reports personal fees from F. Hoffmann-La Roche Ltd as a member of steering committee in transmission study of baloxavir, and grants from Peking Union Medical College Foundation, outside the submitted work. M. Humbert reports grants, personal fees and non-financial support from GlaxoSmithKline, personal fees from AstraZeneca, Novartis, Roche, Sanofi, Teva and Merck, grants and personal fees from Acceleron, Actelion and Bayer, outside the submitted work. M. Shteinberg 
reports grants, personal fees and non-financial support from GSK, grants and personal fees from Novartis, personal fees from Boehringer Ingelheim, AstraZeneca, Kamada, Vertex pharmaceuticals, Teva and Zambon, non-financial support from Actelion and Rafa, outside the submitted work. C.S. Ulrik reports personal fees from AstraZeneca, GSK, Chiesi, Orion Pharma and TEVA, grants and personal fees from Boehringer Ingelheim, Sanofi Genzyme and Novartis, grants from Mundipharma, outside the submitted work. D. Stolz reports grants from AstraZeneca AG, Curetis AG and Boston Scientific, lecture fees from AstraZeneca AG, Novartis AG, GSK AG, Roche AG, Zambon, Pfizer, Schwabe Pharma AG and Vifor AG, outside the submitted work. T. Welte reports grants from German Ministry of Health, German Ministry of Research and Education, DFG, WHO and EU, during the conduct of the study; personal fees for lectures/advisory board work from Roche, AstraZeneca, Boehringer, GSK and Novartis, outside the submitted work. S. Aliberti reports personal fees for advisory board work and research support from Bayer Healthcare, personal fees for lectures from Grifols and Menarini, personal fees for advisory board work from AstraZeneca and ZetaCube Srl, personal fees for advisory board work and lectures from Zambon and GlaxoSmithKline, grants and personal fees advisory board work and lectures from Chiesi and INSMED, grants from Fisher \& Paykel, outside the submitted work. T. Tonia acts as ERS Methodologist. J.D. Chalmers reports grants and personal fees from AstraZeneca, Boehringer Ingelheim, GlaxoSmithKline and Insmed, personal fees from Chiesi, Novartis and Zambon, grants from Gilead Sciences, outside the submitted work. The remaining authors disclose no potential conflicts of interest.

Support statement: Funded by the European Respiratory Society through the COVID-19 Living Guidelines Task Force (2020-14). Funding information for this article has been deposited with the Crossref Funder Registry.

\section{References}

1 Huang C, Wang Y, Li X, et al. Clinical features of patients infected with 2019 novel coronavirus in Wuhan, China. Lancet 2020; 395: 497-506.

2 Ghinai I, McPherson TD, Hunter JC, et al. First known person-to-person transmission of severe acute respiratory syndrome coronavirus 2 (SARS-CoV-2) in the USA. Lancet 2020; 395: 1137-1144.

3 Liang W-H, Guan W-J, Li C-C, et al. Clinical characteristics and outcomes of hospitalised patients with COVID-19 treated in Hubei (epicentre) and outside Hubei (non-epicentre): a nationwide analysis of China. Eur Respir J 2020; 55: 2000562.

4 Guan W-J, Ni Z-Y, Hu Y, et al. Clinical characteristics of coronavirus disease 2019 in China. N Engl J Med 2020; 382: 1708-1720.

5 Deng Y, Liu W, Liu K, et al. Clinical characteristics of fatal and recovered cases of coronavirus disease 2019 (COVID-19) in Wuhan, China: a retrospective study. Chin Med J (Engl) 2020; 133: 1261-1267.

6 Torres Acosta MA, Singer BD. Pathogenesis of COVID-19-induced ARDS: implications for an ageing population. Eur Respir J 2020; 56: 2002049.

7 Mason RJ. Pathogenesis of COVID-19 from a cell biology perspective. Eur Respir J 2020; 55: 2000607.

8 Wu G, Yang P, Xie Y, et al. Development of a clinical decision support system for severity risk prediction and triage of COVID-19 patients at hospital admission: an international multicentre study. Eur Respir J 2020; 56: 2001104

9 Knight SR, Ho A, Pius R, et al. Risk stratification of patients admitted to hospital with Covid-19 using the ISARIC WHO Clinical Characterisation Protocol: development and validation of the 4 C Mortality Score. BMJ 2020; 370: m3339.

10 Docherty AB, Harrison EM, Green CA, et al. Features of 20133 UK patients in hospital with Covid-19 using the ISARIC WHO Clinical Characterisation Protocol: prospective observational cohort study. BMJ 2020; 369: m1985.

11 Du R-H, Liang L-R, Yang C-Q, et al. Predictors of mortality for patients with COVID-19 pneumonia caused by SARS-CoV-2: a prospective cohort study. Eur Respir J 2020; 55: 2000524.

12 Fan G, Tu C, Zhou F, et al. Comparison of severity scores for COVID-19 patients with pneumonia: a retrospective study. Eur Respir J 2020; 56: 2002113.

13 Swann OV, Holden KA, Turtle L, et al. Clinical characteristics of children and young people admitted to hospital with Covid-19 in United Kingdom: prospective multicentre observational cohort study. BMJ 2020; 370: $\mathrm{m} 3249$.

14 Singanayagam A, Hakki S, Dunning J, et al. Community transmission and viral load kinetics of the SARS-CoV-2 delta (B.1.617.2) variant in vaccinated and unvaccinated individuals in the UK: a prospective, longitudinal, cohort study. Lancet Infect Dis 2021; 22: 183-195.

15 Bager P, Wohlfahrt J, Rasmussen M, et al. Hospitalisation associated with SARS-CoV-2 delta variant in Denmark. Lancet Infect Dis 2021; 21: 1351.

16 Karim SSA, Karim QA. Omicron SARS-CoV-2 variant: a new chapter in the COVID-19 pandemic. Lancet 2021; 398: 2126-2128.

17 Kuhlmann C, Mayer CK, Claassen M, et al. Breakthrough infections with SARS-CoV-2 omicron despite mRNA vaccine booster dose. Lancet 2022; 399: 625-626. 

neutralization of SARS-CoV-2 virus-like particles. medRxiv 2022; preprint [https://doi.org/10.1101/2021.12.20. 21268048]. mice and hamsters. Nature 2022; 603: 687-692.

Wolter N, Jassat W, Walaza S, et al. Early assessment of the clinical severity of the SARS-CoV-2 omicron variant in South Africa: a data linkage study. Lancet 2022; 399: 437-446. against SARS-CoV-2: an interim analysis of four randomised controlled trials in Brazil, South Africa, and the UK. Lancet 2021; 397: 99-111.

22 Polack FP, Thomas SJ, Kitchin N, et al. Safety and efficacy of the BNT162b2 mRNA Covid-19 vaccine. $N$ Engl J Med 2020; 383: 2603-2615.

23 Siddiqi HK, Mehra MR. COVID-19 illness in native and immunosuppressed states: a clinical-therapeutic staging proposal. J Heart Lung Transplant 2020; 39: 405-407.

Cevik M, Tate M, Lloyd O, et al. SARS-CoV-2, SARS-CoV, and MERS-CoV viral load dynamics, duration of viral shedding, and infectiousness: a systematic review and meta-analysis. Lancet Microbe 2020; 2: e13-e22. associations with poor prognosis. Nat Med 2020; 26: 1623-1635. Del Valle DM, Kim-Schulze S, Huang H-H, et al. An inflammatory cytokine signature predicts COVID-19 severity and survival. Nat Med 2020; 26: 1636-1643.

27 Mann ER, Menon M, Knight SB, et al. Longitudinal immune profiling reveals key myeloid signatures associated with COVID-19. Sci Immunol 2020; 5: eabd6197.

28 Remy KE, Mazer M, Striker DA, et al. Severe immunosuppression and not a cytokine storm characterize COVID-19 infections. JCI Insight 2020; 5: e140329.

29 Leisman DE, Ronner L, Pinotti R, et al. Cytokine elevation in severe and critical COVID-19: a rapid systematic review, meta-analysis, and comparison with other inflammatory syndromes. Lancet Respir Med 2020; 8: 1233-1244.

30 Chen LYC, Hoiland RL, Stukas S, et al. Confronting the controversy: interleukin-6 and the COVID-19 cytokine storm syndrome. Eur Respir J 2020; 56: 2003006.

31 McElvaney OJ, McEvoy N, McElvaney OF, et al. Characterization of the inflammatory response to severe COVID-19 illness. Am J Respir Crit Care Med 2020; 202: 812-821.

32 Veras FP, Pontelli MC, Silva CM, et al. SARS-CoV-2-triggered neutrophil extracellular traps mediate COVID-19 pathology. J Exp Med 2020; 217: e20201129.

33 Wilk AJ, Rustagi A, Zhao NQ, et al. A single-cell atlas of the peripheral immune response in patients with severe COVID-19. Nat Med 2020; 26: 1070-1076.

Galani I-E, Rovina N, Lampropoulou V, et al. Untuned antiviral immunity in COVID-19 revealed by temporal type I/III interferon patterns and flu comparison. Nat Immunol 2020; 22: 32-40.

35 Wilkinson T, Dixon R, Page C, et al. ACCORD: a multicentre, seamless, phase 2 adaptive randomisation platform study to assess the efficacy and safety of multiple candidate agents for the treatment of COVID-19 in hospitalised patients: a structured summary of a study protocol for a randomise. Trials 2020; 21: 691. WHO Solidarity Trial Consortium, Pan H, Peto R, et al. Repurposed antiviral drugs for COVID-19 - interim WHO SOLIDARITY trial results. medRxiv 2020; preprint [https://doi.org/10.1101/2020.10.15.20209817].

37 Edalatifard M, Akhtari M, Salehi M, et al. Intravenous methylprednisolone pulse as a treatment for hospitalised severe COVID-19 patients: results from a randomised controlled clinical trial. Eur Respir J 2020; 56: 2002808.

38 Mehta P, Porter JC, Manson JJ, et al. Therapeutic blockade of granulocyte macrophage colony-stimulating factor in COVID-19-associated hyperinflammation: challenges and opportunities. Lancet Respir Med 2020; 8: 822-830.

39 Mathioudakis AG, Fally M, Hashad R, et al. COVID-19 clinical trials: unravelling a methodological Gordian knot. Am J Respir Crit Care Med 2020; 202: 635-637.

40 Thomson K, Nachlis H. Emergency use authorizations during the COVID-19 pandemic: lessons from hydroxychloroquine for vaccine authorization and approval. JAMA 2020; 324: 1282-1283.

41 Maor Y, Cohen D, Paran N, et al. Compassionate use of convalescent plasma for treatment of moderate and severe pneumonia in COVID-19 patients and association with IgG antibody levels in donated plasma. EClinicalMedicine 2020; 26: 100525.

42 Paumgartten FJR, Oliveira ACAXD. Off label, compassionate and irrational use of medicines in Covid-19 pandemic, health consequences and ethical issues. Cien Saude Colet 2020; 25: 3413-3419.

43 Rojek AM, Martin GE, Horby PW. Compassionate drug (mis)use during pandemics: lessons for COVID-19 from 2009. BMC Med 2020; 18: 265. 
Brożek JL, Akl EA, Compalati E, et al. Grading quality of evidence and strength of recommendations in clinical practice guidelines part 3 of 3. The GRADE approach to developing recommendations. Allergy 2011; 66: 588-595. outcomes. J Clin Epidemiol 2011; 64: 395-400.

Crichton ML, Goeminne PC, Tuand K, et al. The impact of therapeutics on mortality in hospitalised patients with COVID-19: systematic review and meta-analyses informing the European Respiratory Society living guideline. Eur Respir Rev 2021; 30: 210171. checklist. BMJ 2016; 354: i3507.

Vogel JP, Dowswell T, Lewin S, et al. Developing and applying a 'living guidelines' approach to WHO recommendations on maternal and perinatal health. BMJ Glob Health 2019; 4: e001683.

49 Guyatt GH, Oxman AD, Vist G, et al. GRADE guidelines: 4. Rating the quality of evidence-study limitations (risk of bias). J Clin Epidemiol 2011; 64: 407-415.

Atkins D, Best D, Briss PA, et al. Grading quality of evidence and strength of recommendations. BMJ 2004; 328: 1490.

51 Andrews J, Guyatt G, Oxman AD, et al. GRADE guidelines: 14. Going from evidence to recommendations: the significance and presentation of recommendations. J Clin Epidemiol 2013; 66: 719-725.

Tomazini BM, Maia IS, Cavalcanti AB, et al. Effect of dexamethasone on days alive and ventilator-free in patients with moderate or severe acute respiratory distress syndrome and COVID-19: The CoDEX Randomized Clinical Trial. JAMA 2020; 324: 1307-1316.

53 Horby P, Lim WS, Emberson JR, et al. Dexamethasone in hospitalized patients with Covid-19. N Engl J Med 2021; 384: 693-704.

Sterne JAC, Murthy S, Diaz JV, et al. Association between administration of systemic corticosteroids and mortality among critically ill patients with COVID-19: a meta-analysis. JAMA 2020; 324: 1330-1341.

Angus DC, Derde L, Al-Beidh F, et al. Effect of hydrocortisone on mortality and organ support in patients with severe COVID-19: The REMAP-CAP COVID-19 Corticosteroid Domain Randomized Clinical Trial. JAMA 2020; 324: 1317-1329.

56 Dequin PF, Heming N, Meziani F, et al. Effect of hydrocortisone on 21-day mortality or respiratory support among critically ill patients with COVID-19: a randomized clinical trial. JAMA 2020; 324: 1298-1306.

57 Corral-Gudino L, Bahamonde A, Arnaiz-Revillas F, et al. Methylprednisolone in adults hospitalized with COVID-19 pneumonia: an open-label randomized trial (GLUCOCOVID). Wien Klin Wochenschr 2021; 133: 303-311.

58 Tang $\mathrm{X}$, Feng $\mathrm{Y}-\mathrm{M}, \mathrm{Ni} \mathrm{J}-\mathrm{X}$, et al. Early use of corticosteroid may prolong SARS-CoV-2 shedding in nonintensive care unit patients with COVID-19 pneumonia: a multicenter, single-blind, randomized control trial. Respiration 2021; 100: 116-126.

Jeronimo CMP, Farias MEL, Val FFA, et al. Methylprednisolone as adjunctive therapy for patients hospitalized with coronavirus disease 2019 (COVID-19; Metcovid): a randomized, double-blind, phase IIb, placebo-controlled trial. Clin Infect Dis 2021; 72: e373-e381.

60 Stephen-Victor E, Das M, Karnam A, et al. Potential of regulatory T-cell-based therapies in the management of severe COVID-19. Eur Respir J 2020; 56: 2002182.

61 Ackermann M, Mentzer SJ, Kolb M, et al. Inflammation and intussusceptive angiogenesis in COVID-19: everything in and out of flow. Eur Respir J 2020; 56: 2003147.

62 Taboada M, Rodríguez N, Varela PM, et al. Effect of high versus low dose of dexamethasone on clinical worsening in patients hospitalised with moderate or severe COVID-19 pneumonia: an open-label, randomised clinical trial. Eur Respir J 2021; in press [https://doi.org/10.1183/13993003.02518-2021].

63 Stone JH, Frigault MJ, Serling-Boyd NJ, et al. Efficacy of tocilizumab in patients hospitalized with Covid-19. N Engl J Med 2020; 383: 2333-2344.

64 Rosas IO, Bräu N, Waters M, et al. Tocilizumab in hospitalized patients with severe Covid-19 pneumonia. N Engl J Med 2021; 384: 1503-1516.

65 Salvarani C, Dolci G, Massari M, et al. Effect of tocilizumab vs standard care on clinical worsening in patients hospitalized with COVID-19 pneumonia: a randomized clinical trial. JAMA Intern Med 2020; 181: 24-31.

66 Salama C, Han J, Yau L, et al. Tocilizumab in patients hospitalized with Covid-19 pneumonia. N Engl J Med 2021; 384: 20-30.

67 Veiga VC, Prats JAGG, Farias DLC, et al. Effect of tocilizumab on clinical outcomes at 15 days in patients with severe or critical coronavirus disease 2019: randomised controlled trial. BMJ 2021; 372: n84.

68 Abani O, Abbas A, Abbas F, et al. Tocilizumab in patients admitted to hospital with COVID-19 (RECOVERY): a randomised, controlled, open-label, platform trial. Lancet 2021; 397: 1637-1645.

69 The REMAP-CAP Investigators. Interleukin-6 receptor antagonists in critically ill patients with Covid-19. N Engl J Med 2021; 384: 1491-1502. 

COVID-19 and moderate or severe pneumonia: a randomized clinical trial. JAMA Intern Med 2021; 181: 32-40. Lescure FX, Honda H, Fowler RA, et al. Sarilumab in patients admitted to hospital with severe or critical COVID-19: a randomised, double-blind, placebo-controlled, phase 3 trial. Lancet Respir Med 2021; 9: 522-532.

72 Sivapalasingam S, Lederer $\mathrm{D}$, Bhore $\mathrm{R}$, et al. A randomized placebo-controlled trial of sarilumab in hospitalized patients with Covid-19. medRxiv 2021; preprint [https://doi.org/10.1101/2021.05.13.21256973].

73 Soin AS, Kumar K, Choudhary NS, et al. Tocilizumab plus standard care versus standard care in patients in India with moderate to severe COVID-19-associated cytokine release syndrome (COVINTOC): an open-label, multicentre, randomised, controlled, phase 3 trial. Lancet Respir Med 2021; 9: 511-521.

74 Wang D, Fu B, Peng Z, et al. Tocilizumab in patients with moderate or severe COVID-19: a randomized, controlled, open-label, multicenter trial. Front Med 2021; 15: 486-494.

75 Mulchandani R, Lyngdoh T, Kakkar AK. Deciphering the COVID-19 cytokine storm: systematic review and meta-analysis. Eur J Clin Invest 2021; 51: e13429.

Khan FA, Stewart I, Fabbri L, et al. Systematic review and meta-analysis of anakinra, sarilumab, siltuximab and tocilizumab for COVID-19. Thorax 2021; 76: 907-919.

77 The WHO Rapid Evidence Appraisal for COVID-19 Therapies (REACT) Working Group, Domingo P, Mur I, et al. Association between administration of IL-6 antagonists and mortality among patients hospitalized for COVID-19: a meta-analysis. JAMA 2021; 326: 499-518.

Cavalcanti AB, Zampieri FG, Rosa RG, et al. Hydroxychloroquine with or without azithromycin in mild-to-moderate Covid-19. N Engl J Med 2020; 383: 2041-2052.

79 Mitjà $\mathrm{O}$, Corbacho-Monné $\mathrm{M}$, Ubals $\mathrm{M}$, et al. Hydroxychloroquine for early treatment of adults with mild Covid-19: a randomized-controlled trial. Clin Infect Dis 2020; 73: e4073-e4081.

80 Tang W, Cao Z, Han M, et al. Hydroxychloroquine in patients with mainly mild to moderate coronavirus disease 2019: open label, randomised controlled trial. BMJ 2020; 369: m1849.

81 Skipper CP, Pastick KA, Engen NW, et al. Hydroxychloroquine in nonhospitalized adults with early COVID-19: a randomized trial. Ann Intern Med 2020; 173: 623-631.

82 Horby $\mathrm{P}$, Mafham M, Linsell L, et al. Effect of hydroxychloroquine in hospitalized patients with Covid-19. $N$ Engl J Med 2020; 383: 2030-2040.

83 Abd-Elsalam S, Esmail ES, Khalaf M, et al. Hydroxychloroquine in the treatment of COVID-19: a multicenter randomized controlled study. Am J Trop Med Hyg 2020; 103: 1635-1639.

84 Chen J, Liu D, Liu L, et al. [A pilot study of hydroxychloroquine in treatment of patients with moderate COVID-19]. Zhejiang Da Xue Xue Bao Yi Xue Ban 2020; 49: 215-219.

85 Chen L, Zhang Z, Fu J, et al. Efficacy and safety of chloroquine or hydroxychloroquine in moderate type of COVID-19: a prospective open-label randomized controlled study. medRxiv 2020; preprint [https://doi.org/10. 1101/2020.06.19.20136093].

Chen Z, Hu J, Zhang Z, et al. Efficacy of hydroxychloroquine in patients with COVID-19: results of a randomized clinical trial. medRxiv 2020; preprint [https://doi.org/10.1101/2020.03.22.20040758]. efficacy and tolerability of hydroxychloroquine and a retrospective study in adult patients with mild to moderate coronavirus disease 2019 (COVID-19). PLoS One 2020; 15: e0242763.

88 Ader F, Peiffer-Smadja N, Poissy J, et al. An open-label randomized controlled trial of the effect of lopinavir/ ritonavir, lopinavir/ritonavir plus IFN- $\beta-1 a$ and hydroxychloroquine in hospitalized patients with COVID-19. Clin Microbiol Infect 2021; 27: 1826-1837.

89 Dubée V, Roy P-M, Vielle B, et al. Hydroxychloroquine in mild-to-moderate coronavirus disease 2019: a placebo-controlled double blind trial. Clin Microbiol Infect 2021; 27: 1124-1130.

90 Hernandez-Cardenas C, Thirion-Romero I, Rivera-Martinez NE, et al. Hydroxychloroquine for the treatment of severe respiratory infection by COVID-19: a randomized controlled trial. medRxiv 2021; preprint [https://doi. org/10.1101/2021.02.01.21250371].

91 Lyngbakken MN, Berdal JE, Eskesen A, et al. A pragmatic randomized controlled trial reports lack of efficacy of hydroxychloroquine on coronavirus disease 2019 viral kinetics. Nat Commun 2020; 11: 6-11.

92 Self WH, Semler MW, Leither LM, et al. Effect of hydroxychloroquine on clinical status at 14 days in hospitalized patients with COVID-19: a randomized clinical trial. JAMA 2020; 324: 2165-2176.

93 Ulrich RJ, Troxel AB, Carmody E, et al. Treating COVID-19 with hydroxychloroquine (TEACH): a multicenter, double-blind randomized controlled trial in hospitalized patients. Open Forum Infect Dis 2020; 7: ofaa446.

94 Yao X, Ye F, Zhang $\mathrm{M}$, et al. In vitro antiviral activity and projection of optimized dosing design of hydroxychloroquine for the treatment of severe acute respiratory syndrome coronavirus 2 (SARS-CoV-2). Clin Infect Dis 2020; 71: 732-739.

95 Furtado RHM, Berwanger O, Fonseca HA, et al. Azithromycin in addition to standard of care versus standard of care alone in the treatment of patients admitted to the hospital with severe COVID-19 in Brazil (COALITION II): a randomised clinical trial. Lancet 2020; 396: 959-967. 
Sekhavati E, Jafari F, SeyedAlinaghi S, et al. Safety and effectiveness of azithromycin in patients with COVID-19: an open-label randomised trial. Int J Antimicrob Agents 2020; 56: 106143.

97 Abaleke E, Abbas M, Abbasi S, et al. Azithromycin in patients admitted to hospital with COVID-19 (RECOVERY): a randomised, controlled, open-label, platform trial. Lancet 2021; 397: 605-612.

98 Hinks TS, Cureton L, Knight R, et al. A randomised clinical trial of azithromycin versus standard care in ambulatory COVID-19 - The ATOMIC2 Trial. medRxiv 2021; preprint [https://doi.org/10.1101/2021.04.21. 21255807].

99 Sivapalan P, Ulrik CS, Lapperre TS, et al. Azithromycin and hydroxychloroquine in hospitalised patients with confirmed COVID-19: a randomised double-blinded placebo-controlled trial. Eur Respir J 2022; 59: 2100752. O'Connell TF, Bradley CJ, Abbas AE, et al. Hydroxychloroquine/azithromycin therapy and QT prolongation in hospitalized patients with COVID-19. JACC Clin Electrophysiol 2021; 7: 16-25.

101 Deftereos SG, Giannopoulos G, Vrachatis DA, et al. Effect of colchicine vs standard care on cardiac and inflammatory biomarkers and clinical outcomes in patients hospitalized with coronavirus disease 2019: The GRECCO-19 Randomized Clinical Trial. JAMA Netw Open 2020; 3: e2013136.

102 Lopes MI, Bonjorno LP, Giannini MC, et al. Beneficial effects of colchicine for moderate to severe COVID-19: a randomised, double-blinded, placebo-controlled clinical trial. RMD Open 2021; 7: e001455.

103 RECOVERY Collaborative Group. Colchicine in patients admitted to hospital with COVID-19 (RECOVERY): a randomised, controlled, open-label, platform trial. Lancet Respir Med 2021; 9: 1419-1426.

104 Piantoni S, Colombo E, Airò P, et al. The rationale for the use of colchicine in COVID-19: comments on the letter by Cumhur Cure M et al. Clin Rheumatol 2020; 39: 2489-2490.

105 Leung YY, Yao Hui LL, Kraus VB. Colchicine-Update on mechanisms of action and therapeutic uses. Semin Arthritis Rheum 2015; 45: 341-350.

106 Tardif J-C, Kouz S, Waters DD, et al. Efficacy and safety of low-dose colchicine after myocardial infarction. $N$ Engl J Med 2019; 381: 2497-2505.

107 Scarsi M, Piantoni S, Colombo E, et al. Association between treatment with colchicine and improved survival in a single-centre cohort of adult hospitalised patients with COVID-19 pneumonia and acute respiratory distress syndrome. Ann Rheum Dis 2020; 79: 1286-1289.

108 Diaz R, Orlandini A, Castellana N, et al. Effect of colchicine vs usual care alone on intubation and 28-day mortality in patients hospitalized with COVID-19: a randomized clinical trial. JAMA Netw Open 2021; 4: e2141328.

109 Horby PW, Mafham M, Bell JL, et al. Lopinavir-ritonavir in patients admitted to hospital with COVID-19 (RECOVERY): a randomised, controlled, open-label, platform trial. Lancet 2020; 396: 1345-1352.

110 Cao B, Wang Y, Wen D, et al. A trial of lopinavir-ritonavir in adults hospitalized with severe Covid-19. $N$ Engl J Med 2020; 382: 1787-1799.

111 Yan D, Liu X-Y, Zhu Y-N, et al. Factors associated with prolonged viral shedding and impact of lopinavir/ ritonavir treatment in hospitalised non-critically ill patients with SARS-CoV-2 infection. Eur Respir J 2020; 56: 2000799.

112 Chu CM, Cheng VCC, Hung IFN, et al. Role of lopinavir/ritonavir in the treatment of SARS: initial virological and clinical findings. Thorax 2004; 59: 252-256.

113 Alhumaid S, Mutair AA, Alawi ZA, et al. Efficacy and safety of lopinavir/ritonavir for treatment of COVID-19: a systematic review and meta-analysis. Trop Med Infect Dis 2020; 5: 180.

114 Martínez-López-de-Castro N, Samartín-Ucha M, Paradela-Carreiro A, et al. Real-world prevalence and consequences of potential drug-drug interactions in the first-wave COVID-19 treatments. J Clin Pharm Ther 2020; 46: 724-730.

115 Mahajan L, Singh AP, Gifty. Clinical outcomes of using remdesivir in patients with moderate to severe COVID-19: a prospective randomised study. Indian J Anaesth 2021; 65: S41-S46.

116 Spinner CD, Gottlieb RL, Criner GJ, et al. Effect of remdesivir vs standard care on clinical status at 11 days in patients with moderate COVID-19: a randomized clinical trial. JAMA 2020; 324: 1048-1057.

117 Wang Y, Zhang D, Du G, et al. Remdesivir in adults with severe COVID-19: a randomised, double-blind, placebo-controlled, multicentre trial. Lancet 2020; 395: 1569-1578.

118 Beigel JH, Tomashek KM, Dodd LE, et al. Remdesivir for the treatment of Covid-19 - final report. N Engl J Med 2020; 383: 1813-1826.

119 Agostini ML, Andres EL, Sims AC, et al. Coronavirus susceptibility to the antiviral remdesivir (GS-5734) is mediated by the viral polymerase and the proofreading exoribonuclease. MBio 2018; 9: e00221-18.

120 Sheahan TP, Sims AC, Graham RL, et al. Broad-spectrum antiviral GS-5734 inhibits both epidemic and zoonotic coronaviruses. Sci Transl Med 2017; 9: eaal3653.

121 Goldman JD, Lye DCB, Hui DS, et al. Remdesivir for 5 or 10 days in patients with severe Covid-19. N Engl J Med 2020; 383: 1827-1837.

122 Gottlieb RL, Vaca CE, Paredes R, et al. Early remdesivir to prevent progression to severe Covid-19 in outpatients. N Engl J Med 2021; 386: 305-315. 
123 Davoudi-Monfared E, Rahmani $\mathrm{H}$, Khalili $\mathrm{H}$, et al. A randomized clinical trial of the efficacy and safety of interferon $\beta-1 \mathrm{a}$ in treatment of severe COVID-19. Antimicrob Agents Chemother 2020; 64: e01061-20.

124 Rahmani H, Davoudi-Monfared E, Nourian A, et al. Interferon $\beta$-1b in treatment of severe COVID-19: a randomized clinical trial. Int Immunopharmacol 2020; 88: 106903.

125 Alavi Darazam I, Shokouhi S, Pourhoseingholi MA, et al. Role of interferon therapy in severe COVID-19: the COVIFERON randomized controlled trial. Sci Rep 2021; 11: 8059.

126 Hadjadj J, Yatim N, Barnabei L, et al. Impaired type I interferon activity and inflammatory responses in severe COVID-19 patients. Science 2020; 369: 718-724.

127 Cinatl J, Morgenstern B, Bauer G, et al. Treatment of SARS with human interferons. Lancet 2003; 362: 293-294.

128 Hung IF-N, Lung K-C, Tso EY-K, et al. Triple combination of interferon beta-1b, lopinavir-ritonavir, and ribavirin in the treatment of patients admitted to hospital with COVID-19: an open-label, randomised, phase 2 trial. Lancet 2020; 395: 1695-1704.

129 Monk PD, Marsden RJ, Tear VJ, et al. Safety and efficacy of inhaled nebulised interferon beta-1a (SNG001) for treatment of SARS-CoV-2 infection: a randomised, double-blind, placebo-controlled, phase 2 trial. Lancet Respir Med 2020; 9: 196-206.

130 Lopes RD, de Barros e Silva PGM, Furtado RHM, et al. Therapeutic versus prophylactic anticoagulation for patients admitted to hospital with COVID-19 and elevated D-dimer concentration (ACTION): an open-label, multicentre, randomised, controlled trial. Lancet 2021; 397: 2253-2263.

131 Lemos ACB, do Espírito Santo DA, Salvetti MC, et al. Therapeutic versus prophylactic anticoagulation for severe COVID-19: a randomized phase II clinical trial (HESACOVID). Thromb Res 2020; 196: 359-366.

132 Spyropoulos AC, Goldin M, Giannis D, et al. Efficacy and safety of therapeutic-dose heparin vs standard prophylactic or intermediate-dose heparins for thromboprophylaxis in high-risk hospitalized patients with COVID-19: The HEP-COVID Randomized Clinical Trial. JAMA Intern Med 2021; 181: 1612-1620.

133 Goligher D, Bradbury D, Berry W, et al. Therapeutic anticoagulation with heparin in critically ill patients with Covid-19. N Engl J Med 2021; 385: 777-789.

134 The ATTACC A-4a, and RECAMP-CAP Investigators. Therapeutic anticoagulation with heparin in noncritically ill patients with Covid-19. N Engl J Med 2021; 385: 790-802.

135 Jiménez D, García-Sanchez A, Rali P, et al. Incidence of VTE and bleeding among hospitalized patients with coronavirus disease 2019: a systematic review and meta-analysis. Chest 2020; 159: 1182-1196.

136 Si-Mohamed S, Chebib N, Sigovan M, et al. In vivo demonstration of pulmonary microvascular involvement in COVID-19 using dual-energy computed tomography. Eur Respir J 2020; 56: 2002608.

137 Price LC, McCabe C, Garfield B, et al. Thrombosis and COVID-19 pneumonia: the clot thickens! Eur Respir J 2020; 56: 2001608.

138 Ospina-Tascón GA, Calderón-Tapia LE, García AF, et al. Effect of high-flow oxygen therapy vs conventional oxygen therapy on invasive mechanical ventilation and clinical recovery in patients with severe COVID-19: a randomized clinical trial. JAMA 2021; 326: 2161-2171.

139 Teng XB, Shen Y, Han MF, et al. The value of high-flow nasal cannula oxygen therapy in treating novel coronavirus pneumonia. Eur J Clin Invest 2021; 51: e13435.

140 Perkins GD, Ji C, Connolly BA, et al. An adaptive randomized controlled trial of non-invasive respiratory strategies in acute respiratory failure patients with COVID-19. medRxiv 2021; preprint [https://doi.org/10. 1101/2021.08.02.21261379].

141 Ferreyro BL, Angriman F, Munshi L, et al. Association of noninvasive oxygenation strategies with all-cause mortality in adults with acute hypoxemic respiratory failure: a systematic review and meta-analysis. JAMA 2020; 324: 57-67.

142 Richards-Belle A, Orzechowska I, Gould DW, et al. COVID-19 in critical care: epidemiology of the first epidemic wave across England, Wales and Northern Ireland. Intensive Care Med 2020; 46: 2035-2047.

143 Winslow RL, Zhou J, Windle EF, et al. SARS-CoV-2 environmental contamination from hospitalised patients with COVID-19 receiving aerosol-generating procedures. Thorax 2021; 77: 259-267.

144 Hamilton FW, Gregson FKA, Arnold DT, et al. Aerosol emission from the respiratory tract: an analysis of aerosol generation from oxygen delivery systems. Thorax 2021; 77: 276-282.

145 Li J, Fink JB, Ehrmann S. High-flow nasal cannula for COVID-19 patients: low risk of bio-aerosol dispersion. Eur Respir J 2020; 55: 2000892.

$146 \mathrm{Ng} \mathrm{Z}$, Tay WC, Ho CHB. Rationale and significance of patient selection in awake prone positioning for COVID-19 pneumonia. Eur Respir J 2020; 56: 2002571.

147 Reddy MP, Subramaniam A, Lim ZJ, et al. Prone positioning of non-intubated patients with COVID-19 - a systematic review and meta-analysis. medRxiv 2020; preprint [https://doi.org/10.1101/2020.10.12.20211748].

148 Coppo A, Bellani G, Winterton D, et al. Feasibility and physiological effects of prone positioning in non-intubated patients with acute respiratory failure due to COVID-19 (PRON-COVID): a prospective cohort study. Lancet Respir Med 2020; 8: 765-774. 
149 Huang C-F, Tay CK, Zhuang Y-F, et al. Rationale and significance of patient selection in awake prone positioning for COVID-19 pneumonia. Eur Respir J 2020; 56: 2002173.

150 Cheng Y, Wong R, Soo YOY, et al. Use of convalescent plasma therapy in SARS patients in Hong Kong. Eur J Clin Microbiol Infect Dis 2005; 24: 44-46.

151 Joyner MJ, Carter RE, Senefeld JW, et al. Convalescent plasma antibody levels and the risk of death from Covid-19. N Engl J Med 2021; 384: 1015-1027.

152 Abani O, Abbas A, Abbas F, et al. Convalescent plasma in patients admitted to hospital with COVID-19 (RECOVERY): a randomised controlled, open-label, platform trial. Lancet 2021; 397: 2049-2059.

153 Libster R, Marc GP, Wappner D, et al. Early high-titer plasma therapy to prevent severe Covid-19 in older adults. N Engl J Med 2021; 384: 610-618.

154 Sekine L, Arns B, Fabro BR, et al. Convalescent plasma for COVID-19 in hospitalised patients: an open-label, randomised clinical trial. Eur Respir J 2022; 59: 2101471.

155 Simonovich VA, Burgos Pratx LD, Scibona P. A randomized trial of convalescent plasma in Covid-19 severe pneumonia. N Engl J Med 2020; 384: 619-629.

156 Gharbharan A, Jordans CCE, GeurtsvanKessel C, et al. Effects of potent neutralizing antibodies from convalescent plasma in patients hospitalized for severe SARS-CoV-2 infection. Nat Commun 2021; 12: 3189.

157 Bennett-Guerrero E, Romeiser JL, Talbot LR, et al. Severe acute respiratory syndrome coronavirus 2 convalescent plasma versus standard plasma in coronavirus disease 2019 infected hospitalized patients in New York: a double-blind randomized trial. Crit Care Med 2021; 49: 1015-1025.

158 Abolghasemi H, Eshghi P, Cheraghali AM, et al. Clinical efficacy of convalescent plasma for treatment of COVID-19 infections: results of a multicenter clinical study. Transfus Apher Sci 2020; 59: 102875.

159 Li L, Zhang W, Hu Y, et al. Effect of convalescent plasma therapy on time to clinical improvement in patients with severe and life-threatening COVID-19: a randomized clinical trial. JAMA 2020; 324: 460-470.

160 AlQahtani M, Abdulrahman A, Almadani A, et al. Randomized controlled trial of convalescent plasma therapy against standard therapy in patients with severe COVID-19 disease. Sci Rep 2021; 11: 9927.

161 O'Donnell MR, Grinsztejn B, Cummings MJ, et al. A randomized double-blind controlled trial of convalescent plasma in adults with severe COVID-19. J Clin Invest 2021; 131: e150646.

162 Kirenga B, Byakika-Kibwika P, Muttamba W, et al. Efficacy of convalescent plasma for treatment of COVID-19 in Uganda. BMJ Open Respir Res 2021; 8: e001017.

163 Agarwal A, Mukherjee A, Kumar G, et al. Convalescent plasma in the management of moderate Covid-19 in adults in India: open label phase II multicentre randomised controlled trial (PLACID Trial). BMJ 2020; 371: m3939.

164 Pouladzadeh M, Safdarian M, Eshghi P, et al. A randomized clinical trial evaluating the immunomodulatory effect of convalescent plasma on COVID-19-related cytokine storm. Intern Emerg Med 2021; 16: 2181-2191.

165 Körper S, Weiss M, Zickler D, et al. High dose convalescent plasma in COVID-19: results from the randomized trial CAPSID. medRxiv 2021; preprint [https://doi.org/10.1101/2021.05.10.21256192].

166 Avendaño-Solà C, Ramos-Martínez A, Muñez-Rubio E, et al. Convalescent plasma for COVID-19: a multicenter, randomized clinical trial. medRxiv 2020; preprint [https://doi.org/10.1101/2020.08.26.20182444].

167 Rasheed AM, Fatak DF, Hashim HA, et al. The therapeutic effectiveness of convalescent plasma therapy on treating COVID-19 patients residing in respiratory care units in Baghdad, Iraq. medRxiv 2020; preprint [https://doi.org/10.1101/2020.06.24.20121905].

168 The CONCOR-I Study Group, CORCOR-I Writing committee, Bégin P, et al. Convalescent plasma for hospitalized patients with COVID-19 and the effect of plasma antibodies: a randomized controlled, open-label trial. medRxiv 2021; preprint [https://doi.org/10.1101/2021.06.29.21259427].

169 RECOVERY Collaborative Group, Horby PW, Mafham M, et al. Casirivimab and imdevimab in patients admitted to hospital with COVID-19 (RECOVERY): a randomised, controlled, open-label, platform trial. medRxiv 2021; preprint [https://doi.org/10.1101/2021.06.15.21258542].

170 Lundgren JD, Grund B, Barkaus-Kas CE, et al. A neutralizing monoclonal antibody for hospitalized patients with Covid-19. N Engl J Med 2021; 384: 905-919.

171 Caricchio R, Abbate A, Gordeev I, et al. Effect of canakinumab vs placebo on survival without invasive mechanical ventilation in patients hospitalized with severe COVID-19: a randomized clinical trial. JAMA 2021; 326: 230-239.

172 Mariette X, Hermine O, Resche-Rigon M, et al. Effect of anakinra versus usual care in adults in hospital with COVID-19 and mild-to-moderate pneumonia (CORIMUNO-ANA-1): a randomised controlled trial. Lancet Respir Med 2021; 9: 295-304.

173 Kyriazopoulou E, Poulakou G, Milionis H, et al. Early treatment of COVID-19 with anakinra guided by soluble urokinase plasminogen receptor plasma levels: a double-blind, randomized controlled phase 3 trial. Nat Med 2021; 27: 1752-1760.

174 The REMAP-CAP Investigators, Derde LPG. Effectiveness of tocilizumab, sarilumab, and anakinra for critically ill patients with COVID-19 The REMAP-CAP COVID-19 Immune Modulation Therapy Domain Randomized Clinical Trial. medRxiv 2021; preprint [https://doi.org/10.1101/2021.06.18.21259133]. 
175 Schwartz DM, Kanno Y, Villarino A, et al. JAK inhibition as a therapeutic strategy for immune and inflammatory diseases. Nat Rev Drug Discov 2017; 16: 843-862.

176 Luo W, Li YX, Jiang LJ, et al. Targeting JAK-STAT signaling to control cytokine release syndrome in COVID-19. Trends Pharmacol Sci 2020; 41: 531-543.

177 Marconi VC, Ramanan AV, de Bono S, et al. Efficacy and safety of baricitinib for the treatment of hospitalised adults with COVID-19 (COV-BARRIER): a randomised, double-blind, parallel-group, placebo-controlled phase 3 trial. Lancet Respir Med 2021; 9: 1407-1418.

178 Kalil A, Patterson TF, Mehta KM, et al. Baricitinib plus remdesivir for hospitalized adults with Covid-19. N Engl J Med 2021; 384: 795-807.

179 Cao Y, Wei J, Zou L, et al. Ruxolitinib in treatment of severe coronavirus disease 2019 (COVID-19): a multicenter, single-blind, randomized controlled trial. J Allergy Clin Immunol 2020; 146: 137-146.e3.

180 Guimarães PO, Quirk D, Furtado RH, et al. Tofacitinib in patients hospitalized with Covid-19 pneumonia. N Engl J Med 2021; 385: 406-415.

181 Ely EW, Ramanan AV, Kartman CE, et al. Baricitinib plus standard of care for hospitalised adults with COVID-19 on invasive mechanical ventilation or extracorporeal membrane oxygenation: results of a randomised, placebo-controlled trial. medRxiv 2021; preprint [https://doi.org/10.1101/2021.10.11.21263897].

182 RECOVERY Collaborative Group, Horby PW, Emberson JR, et al. Baricitinib in patients admitted to hospital with COVID-19 (RECOVERY): a randomised, controlled, open-label, platform trial and updated meta-analysis. medRxiv 2022; preprint [https://doi.org/10.1101/2022.03.02.22271623].

183 Singh D, Bogus M, Moskalenko V, et al. A phase 2 multiple ascending dose study of the inhaled pan-JAK inhibitor nezulcitinib (TD-0903) in severe COVID-19. Eur Respir J 2021; 58: 2100673.

184 Zhang X, Shang L, Fan G-H, et al. The efficacy and safety of Janus kinase (JAK) inhibitors for COVID-19 patients: a living systematic review and meta-analysis. Front Med 2022; 8: 800492. 